\title{
NOUVELLES INSCRIPTIONS DE LYON
}

\author{
par Jean-François REYNAUD, Benoît HELLY et Marcel LE GLAY
}

Des fouilles de sauvetange, entreprises à Lyon all début du printemps de 1980 , ont livré des inscriptions qüil parait utile de faire connaitre sans tarder. Elle proviennent en effet de deux nécropoles qui, dans la phase la plus tardive de leur histoire, ont réutilisé, entre autres, des pierres inscrites, "empruntées" à des tombes plus anciennes. Les inscriptions sont donc sans relation directe avec les cimetières où elles ont été retrouvées. Tout au plus fournissent-elles, dans la mesure où elles sont datables, un lerminus posl quem, à condition de tenir compte d'une période assez longue entre le temps de leur gravure comme epitaphes et le moment de leur remploi. C"est pourquoi elles peuvent ètre présentées des maintenant, sans attendre la publication complète des fouilles et du matériel qu'elles ont livré1. Avant l'étude des textes, on se contentera de brèves indications sur les nécropoles elles-mêmes. Lat première est située sur le llane de la colline de Saint-Irénée, à quelques centaines de mètres des églises de Saint-Just, mises au jour dans les années 1970-19722. La deuxième se trouve dans la partie basse de la rur Pierre-Aurlẹy, du côte de liest, en contrebas de la colline de Fourvierese.

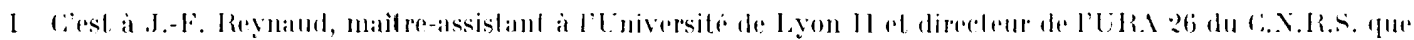

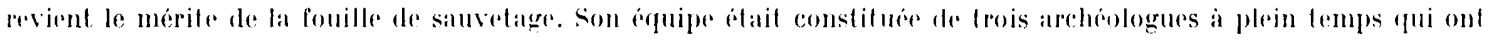

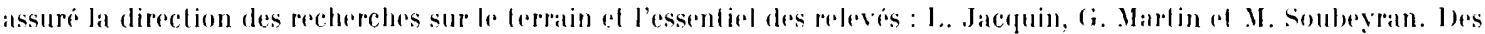

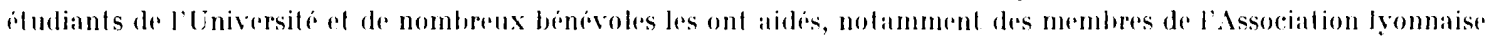
pour le sauvelage des sites archéologigues médievaux, sous la direction de (i. Franck, ainsi que des membres du Groupe lyounais de recherches archelogiques gallo-romaines, diriges par I.. Blanchard. I'ne couverture photo-

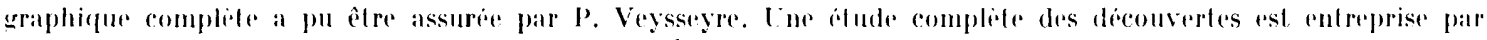

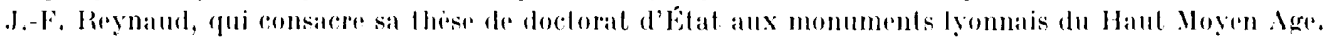

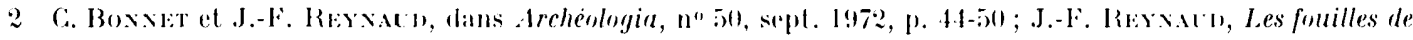
saurelage de l'église Sarint-Just el du groupe épiscopal de Lyon, dans Comples rendus de l'Academie des Inscriphions et

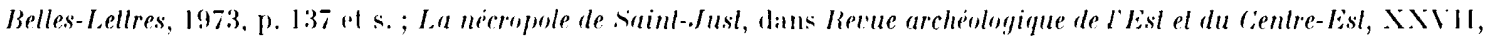

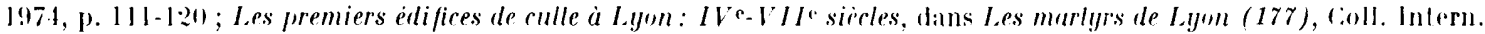

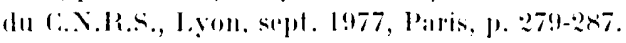

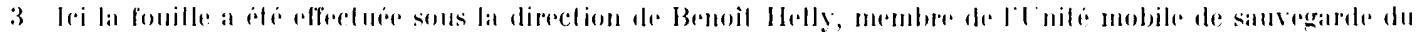

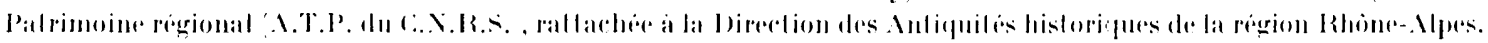

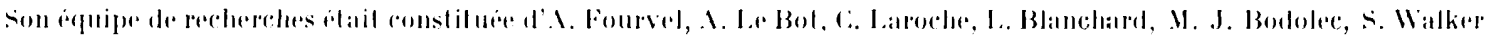

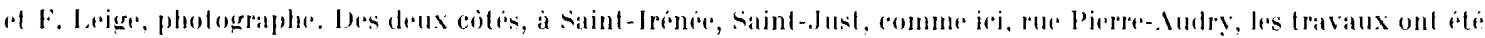

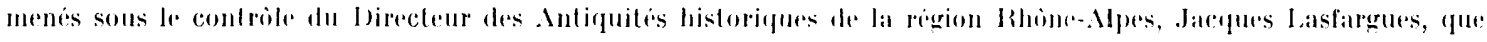
tous les fouillerurs, comme les anteurs de ced article, sont heureux de remercier. 


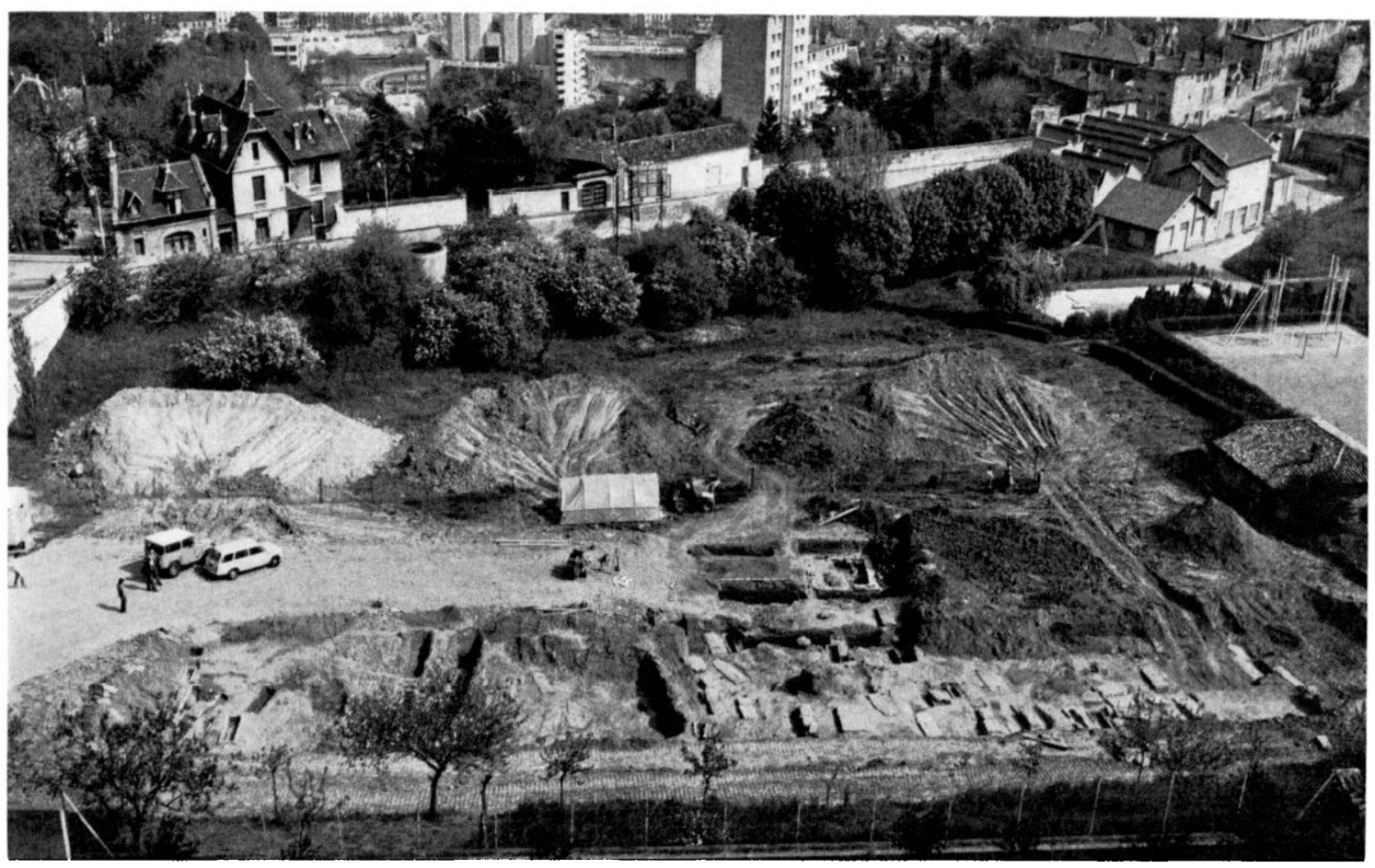

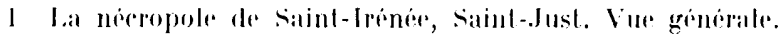

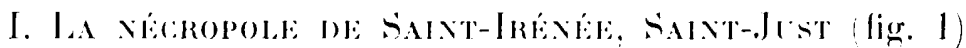

\section{A. Les décounerles archéolngiques.}

A loceasion de lourerture diun rhantier de construetion, il a ete possible, en 70 jours de travail ininterrompu. de repérer et parfois de fouiller quelque 187 sépultures. Le quartier en forte pente au pied de la colline de saint-Irénée a servi trés tôt de nécropole, comme an temoignent une inhumation datee de la fin du ser siecle par son mobilier et les remplois nombreux de monuments funéraires romains dans la nécropole tardive. Aux re-re sièces sy developpa une nécropole chrétienne importante, qui faisait sans doute partie de celle de siant-Irénée a l'ouest et probablement aussi du moins en était-elle

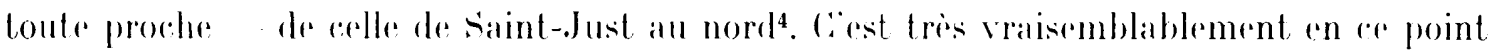
préeis que doit se situer le passige de la voir de Narbonnaise, et non pas plus a l'ouest, romme on l'a ceru, all sommel de Saint-Irénée. En témoignerait notamment l'alignement de deux memoriae.

La partie explorée de la nécropole comprenait une grande variété de Lombes (lig. :2). Outre deux (peut-ètre trois) mausolées abritant des tombes importantes, on comptr un assez grand nombre de sépultures en pleine terre, les unes aver bordure de galets, d'aulres romportant un cercureil de bois. La miljorite des tombes sont des sarcophagers, soit a currs

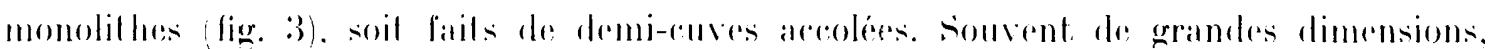




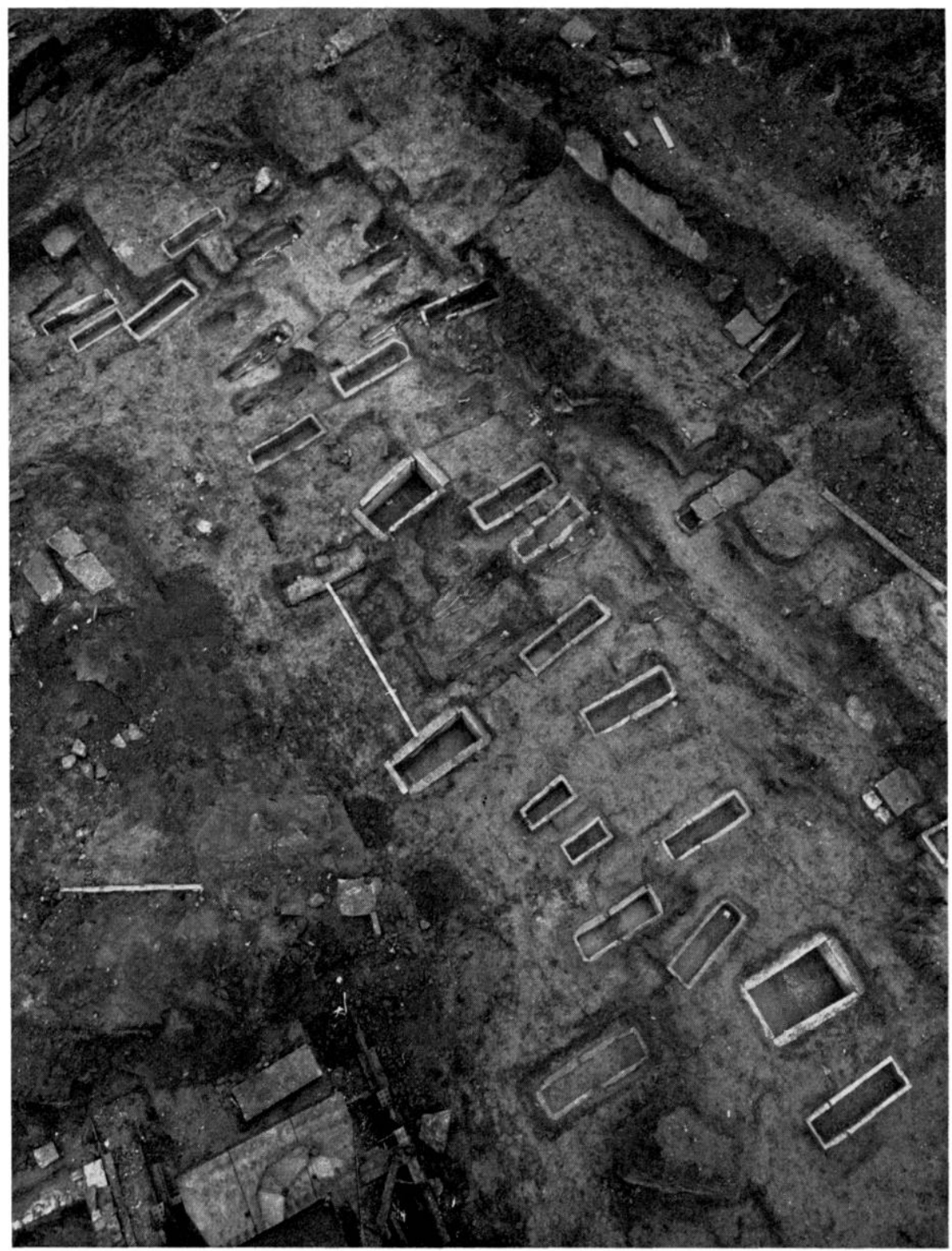

2 La nécropole de Saint-Irinèe, Saint-Just : zone sul.

parfois énormes lals ceux qui portent les inscriptions 1 et 2 . ils sont en général tailless dans des blocs de choin, calcaire du Bas-Bugey, quelques-uns dans un ralcaire plus tendre. Maris il y a aussi des tombes construites en tuiles, ou en pierres posées de rhant, constituanl des sortes de caissons. llaulres, enfin. bisomes ou monoplacese sont des raveaux batis an dalles de choin assez epaisses. de grandes dimensions pour les longs coldes. plus pet ites

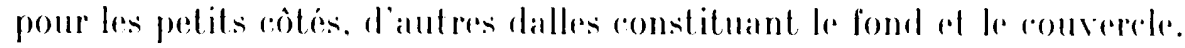

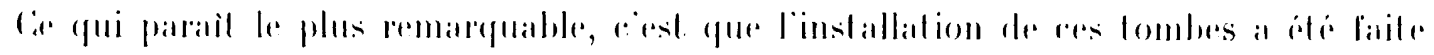

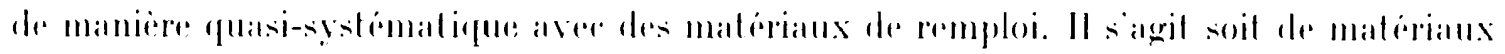
provernant de monuments publies antigues, notamment des blors de grese des replo-

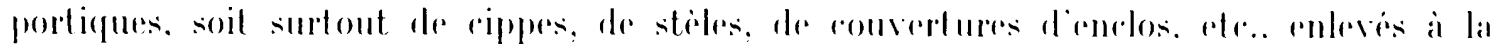
nereropole paitenne. 


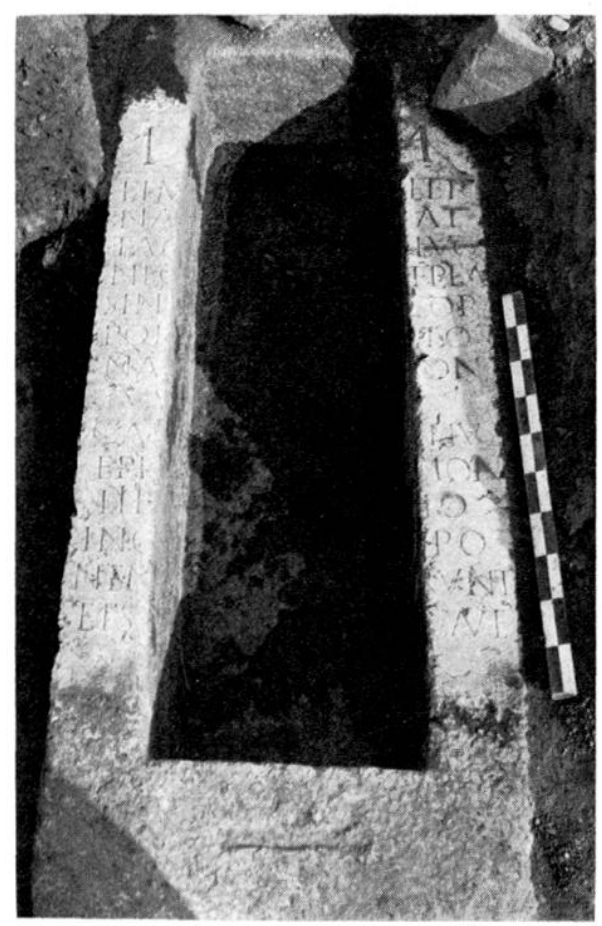

3 Bloc inserit texte no li, relaille an cure de sarcophage.

si lon tient compte des divers comements dates par la ceramique, les monnaies et le maleriel motallique, far comparaison aussi aver dautres sites, on constale que, mises a part quelques rares tombes à incineration. la plupart des sepultures nouvellement deeouvertes sont datables des ave at ve siecles. Et c'est sans doute à ces lieux que correspond la description faite par Sieloine Apollinaire (Ep., 17, rh. I) en 469), à loceasion äun pelerinage à saint-Just. Ia tombe de lancien consul syagrius, qu il signale a une portec de fleche de l'eglise, soit exactement à l'emplacement de la fouille, devait ressembler aux mausolés qui ont ete retrouves.

J.F. R.

\section{Les inscriplions.}

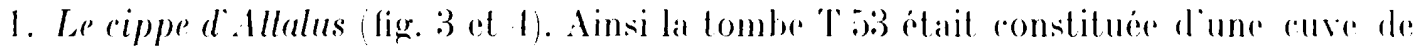
sareophage monolithe creusée dans la partie inserite diun cippe funeraire, et dont le couvercle etait un énorme bloc d'architecture emprunte certainement à un monument important. Ia calue mesure 2.17 m de long sur $0.95 \mathrm{~g}$ m de large et 0.77 de haut. lin la creusant dans le cippe ouvert dime inseription funéraire. on a fait disparaitre la plus grande partire rn fail toute la partie centrale du texte. Mais de ce dernier, qui romportait seize lignes, subsistent, all debut re a la fin dre chaque ligne, deux à trobs lettres, exceptionnellement quatre. les paures restes suffisent pour restituer la majenre partie du lexte initial, lantòt de faceon certaine tantöt de manière plus conjecturale. Certes. fer document lyonnais na a pas le mème interèt historique que l’inseription rélebre de Bruhl qui. retrouvée 
dans un etat comparable de conservation, contenat le cursus de Pertinax alvant son areession a lempires ; il vient cependant enrichir notre connaissane de la sociéte lyonnaise.

Les deux premières lignes ne présentent pas de difficultes. Nous y trouvons l'invocation habituelle aux dieux Mànes. suivie de la formule qui apparait a lyon et dans la région lyonnaise au début du I $^{\mathrm{e}}$ s. pour s'y répandre très largement après 140 : el memoriae a eler|na ej. Le début d'un .I a la ligne 2 impose cette restitution, de préférence à quieli aelernae ou securitali aelernae, qui constituent des variantes de la première formuler. suivent normalement les noms du défunt, qui ont completement disparu au cours de lopération de taille de la cuve du sareophage, sauf te début du cognomen : (point séparatif) A' | TAL, qu'on complétera aisément en Allallus?, surnom bien connu à Rome, an Italie at dans les provinces, rare à Lyon mème où l'on rencontre plus sourent .llicius qui .lllalus, mais qui est tout de mème attesté. Cäest -.. on s'en souvient - le nom d'un des compagnons de martyre de Blandine en 177, citoyen romain qui. selon le texte de la lettre sur les marlyrs rapporté par Eusèbe, parlait latin bien qu'homme de Pergame et qui fut jete aux bètes, au lieu d’ètre décapité selon la décision impériale, bien que ciloyen. Quant au cas, notons qu'en général cest le génitif qui suit D) (is) M(anibus) el memoriar aelernae ${ }^{8}$. Mais on trouve aussi quelquefois le datif' at mème le nominatifle. Rertenons comme le plus vraisemblable le génitif Altal $i$.

Viennent ensuite plusieurs renseignements d'orde socio-professionnel, romme il est fréquent sur les stèles et cippes funeraires lyonnais, qui fournissent ainsi un assez bon tableau de la vie économique et sociale à Lugdunum. Tout de suite après la nomenclature du défunt, la présence à la fin de la ligne 4 de LVC invite à restituer (se)uir Aug(uslalis) Lug(duni). Le nom d'Attalus vient ainsi sajouter aux quelque soixante-dix mentions de seuiri Auguslales de Lyon, dont R. Duthoy a dressé récemment la list ${ }^{11}$. Sans entrer dans le détail des discussions sur la nature et la fonction de l'augustalite, on peut dire

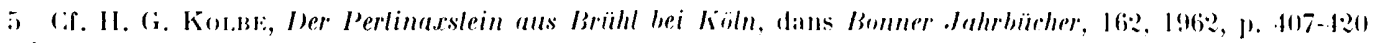

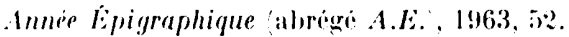

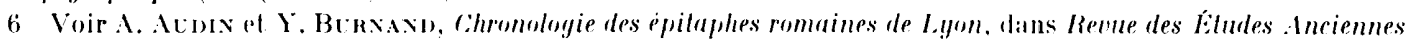
(abrege R.E.A. , I.XI, 1959!, p. 325.

7 Voir Les marlyrs de L.yon (177), en particulier la communication de (;. W. Bow l:Rsock, Les eglises de Lyon el de Vienne: relalions alec l.Asie, p. 249-25); qui "sans nier l'existence à l,yon d’un groupe de chribliens helleniques, pense, quil n'y a pas de base sur laquelle on puisse construire une hypothise fuant aux relations speciales entre les

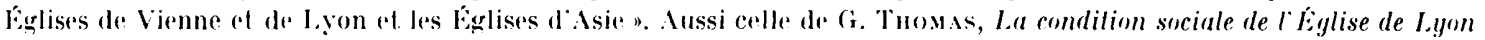
en 177, p. 93-106, en particulier p. 9x-99. Ce qui ne conduit nublement a nier non plus l'existence à lanylunum de nombreux (Orientaux, marchands at artisans : cf. A. Arow, Ciens de Lugdunum, dans . Velanges .M. Renard, II, coll. Latomus, Bruxelles, 1969, p. 14-56; J. Roctis, Aspects économiques de L.yon anlique, dans Les marlyrs de L.yon (177),

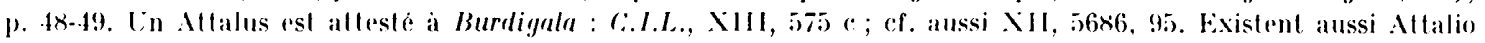
'C.I.L., XII, 3433, el Allalis 750, mais Mtlalus est plus répandu dans le monde romain : ef. Jes Indices des I.l...5.

8 l'ar ex. C..l.L., XIII, 1986, 1995), 2036, 2037, otc.

9 Par ex. C.I.L., XIII, $1 \times 17,1 \times 22,1 \times 51,1860,1 \times 9 \%$, elc.

10 l'ar ex. G.I.L., XIII, IX4I.

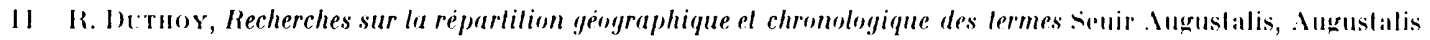

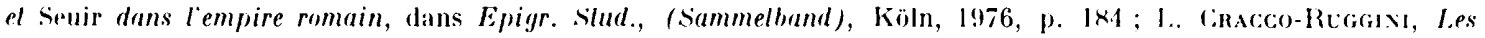
structures de la sociéte el de léconomie lyonnaise an IIe siérle, par rapport à la politique locale el impériale, dans les

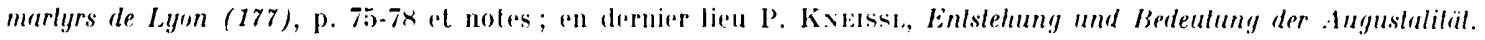

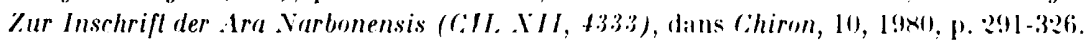




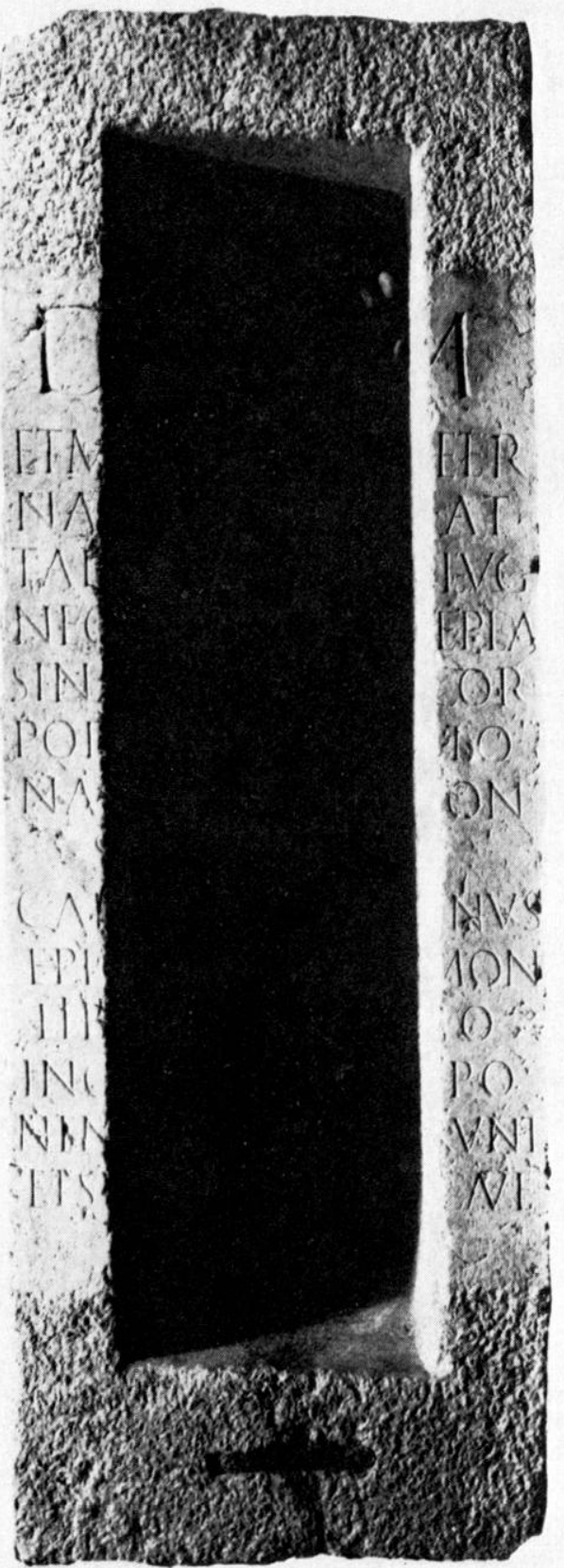

1 J.e cippe d'.1llalus.

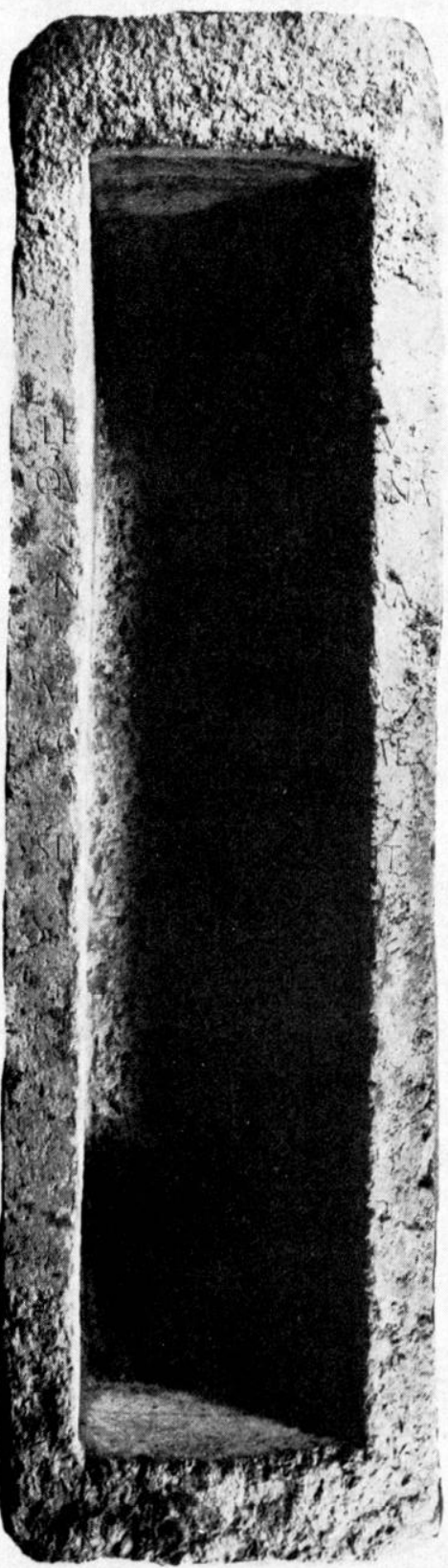

5 Cippe :monymte. 


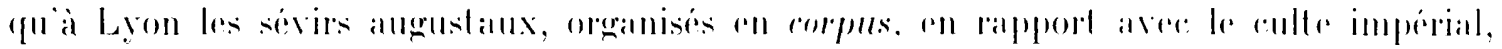

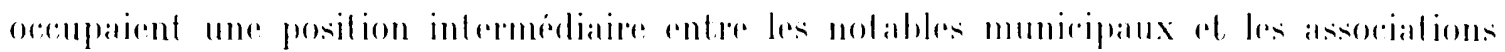
professionnelles.

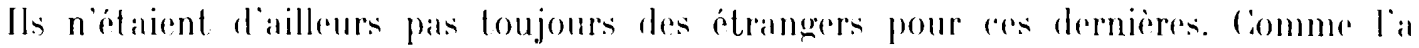

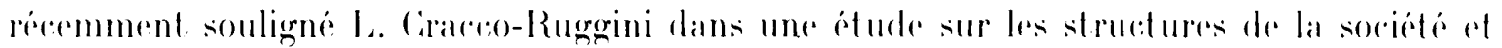

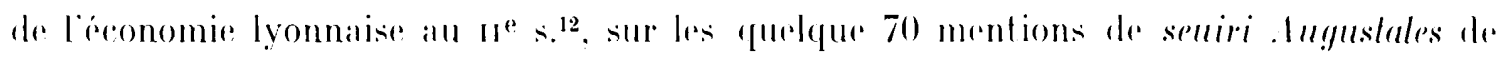

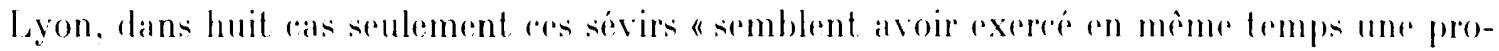
fession lurative organisée en assoceiation 》. Il nous fandra revenir sur aedfe question. Sotons seulement pour le moment que notre Attale parailt quant a lui. aroir rxeree trois: professions differentes, dont une all moins ast presentere romme oreranisore en association.

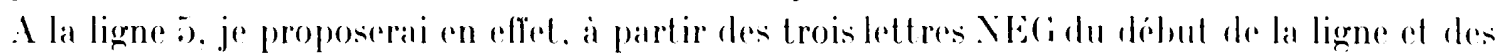
quatre lettres de la fin de la mème ligne qui forment un lout aree les deux premieress

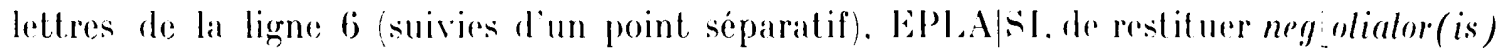

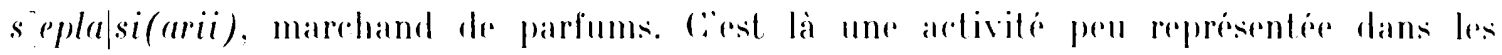

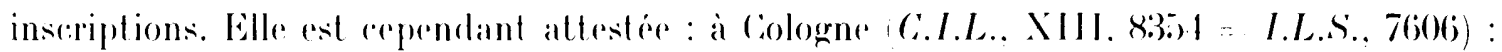
Sers(ao) IIaparo|nio Iuslino | negolialo|ri seplasia|rio fralres | fac(iendum) cur(anerunl) :

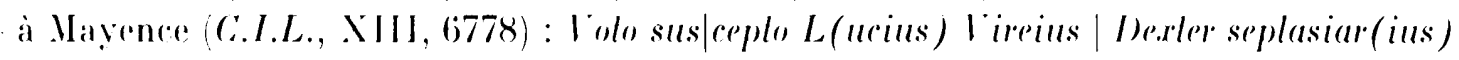

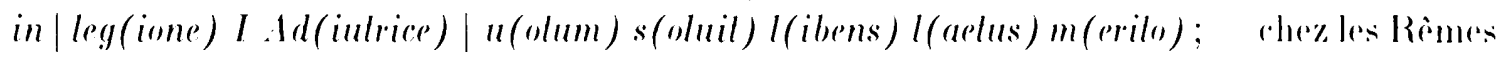

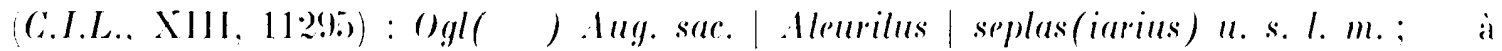
Narbonne (C.I.L., XII, 5971): - - Tertius seplasiar ius : a Florence (C.I.L... XI. $16: 21=$ I.L.S., 7607) : seplasiarius negolians.

Je reviendrai sur re negoce un peu plus loin. Apres aetle mention, pour le moment unique a J yon, la ligne 6 contient une deuxieme indiation professionnelle : X nous invite à restiluer $n$ lanlae, soit Rhod(anici) ou Rhodan(ici) eomme l. Primius Seroundus (C.I.L...

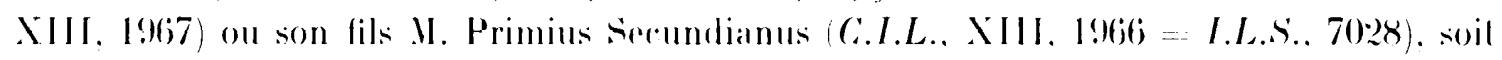
Arar(ici) romme Toutius Incitatus (C..I.L.. XIII. 1972). A la fin re la mème ligne 6 , on

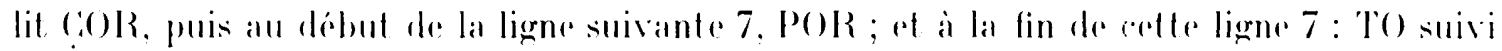

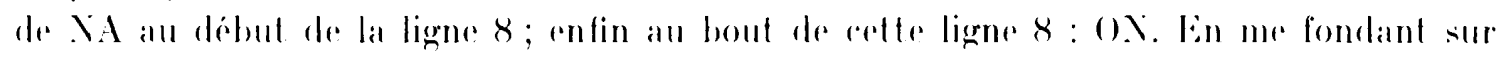
plusieurs textes lyonnais ${ }^{13}$. je propose do restituer : con|por ali inler cen lo|na rios Lugud(uni) coml!sistenles, a l̋instar de II. Primius sorundianus. seuir Aug(uslalis) c. C.C.. Aug. Lug.. naula Rhodan(icus) Arare nanigans, corporal(us) inler fabros lign(uarios) Lug(uduni) consisl(entes), negot(ians) muriar(ius), c'est-a-dire naule du lihòne naviguant sur la saòne, charpentier honoraire. remme traduil .J. Rougerse et marchand de saumures. Notre Allale aurait done elé, quant à lui. membre honoraire de l'assoriation

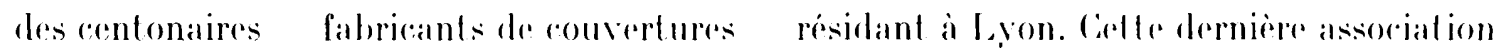
y est hien attesteris.

1: lar. cil., j. 76 in mole 1

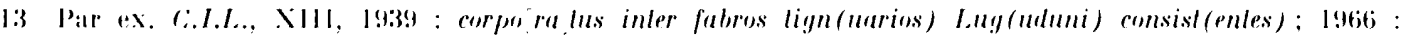
corporal(us) inler fabros lign(uarios) Lu!g(uduni) consisl(enles); 19!x : corporalus inler utriclar (ios) Lu!g(uluni) ronsistentium sie pour consistenles, ; :039 : incorporatus inler ulriclur(ios) Luy(uduni) cons(istentes).

14 J. Rocali, Asperls ecomomiques..., dans Les marlyrs de L.yon (177), p. B.

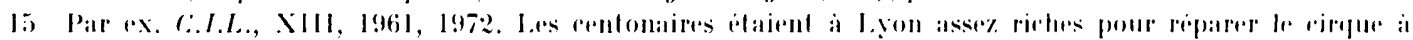

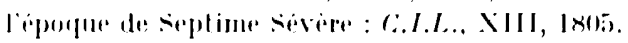


Sous arrivons ainsi à la deuxieme partie du nouveau texte, qui commence à la ligne 10 par le's noms alu nominalif des dédicants qui ont voulu honorer la mémoire d'Attalus. Comme pour ce dernier, leur nomenchature est quasi impossible à restituer. On peut penser à C(aius) AI ou AL[_...], ou plutòt à Cal[limorphus jou Cal[lislio] ou Cae[sonius] (comme nomen) et à TAppia nus par exemple; puis à la ligne 11 à Epiclelus ou Epic arpius: ou Epig[onus]. suivi de Lrle tmon ou [I)a mon ou L'alae mon, tous noms d'affranchis attestés à Lyon ${ }^{16}$. Cair la seule chose sùre, c'est précisément qu il s'agit de lib(erli), comme l’indique le debut de la ligne 12, de liberli qui veulent honorer leur patron. Lequel est qualifié de la manière la plus courante qui soit' ${ }^{17}$ : [palrono opli jmo inc [omparabiliq(ue)]. Et pour finir, vient la formule, aisée à restituer d'après les quelques lettres qui subsistent à la lin et au début des lignes 13, 14 et 15: po|nen|dum curauer unl | el s[ub ascia dedi carule [runl].

Hormis le prénom at le gentilice du défunt qui nous échappent complètement at las noms des dédicants qui ne sont pour deux d'entre eux qu'lypothétiques, on peut don: retrouver le texte original malmené par un tailleur de pierre de basse époque :

I)(is) (ascia?) M(anibus) | el mlemoriae a eler|nale--] Altal[i (se)uir(i) Aug(ustalis)! Lug(uduni), | negolialor(is) sjepla|si(arii), nlaulae Rhodan(ici) ou Arar(ici), cor|por ali inler cen lon|na[rios Lugud(uni) con|s|islentes, | Cal! - -. -nus, | Epiçlelus?, Arle" mon | lib(erti) |palrono oplimo | inc|omparabiliq(ur)?

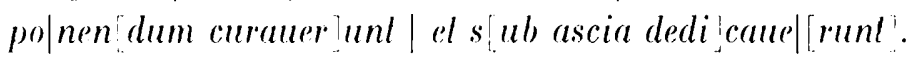

Ce texte présente un triple intérèt. Cielui d’abort d'ajouter une pièce au dossier dójà très fourni - on l'a viu des seniri luguslales de lyon. Je rappelle que sur un total d'environ 70 inscriptions, presque toutes funéraires, qui nomment des sévirs augustaux, 62 proviennent de Lyon mème, alors que huit seulement ont eté retrouvées dans la province de Lyonnaise; encore certaines appartiennent-elles a des Lyonnais. Linstitution sévirale apparait done d'une importance particulière à Lugdunum, où un texte fait connaître en outre des fralres dugustales, une confrérie dont on ignore les statuts, l'organisation et les relations avec le corps des sevirs augustaux, mais dont on peut penser que, comme ces derniers, elle avait pour raison d'être d'honorer l'empereur ${ }^{18}$. Ajoutons que par sal date - nous avons vu que la formule initiale D. M. el memoriae aelernae situe ce nouveau texte dans la seconde moitie du I $^{\mathrm{e}} \mathrm{s}$. - celui-ci s'insère parfaitement dans la série connue. On

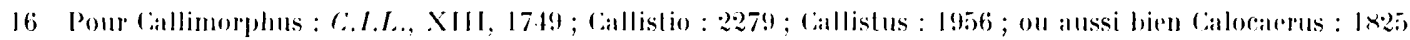

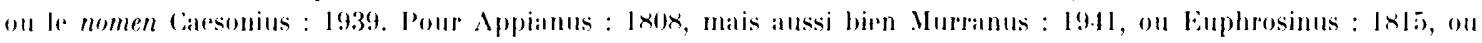

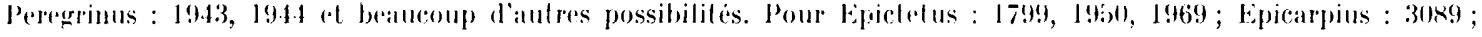

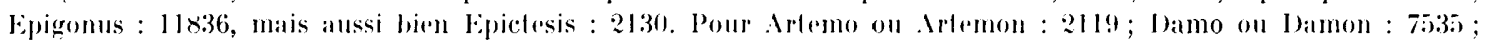
Palatemon : 1942, 2088

17 Palronus oplimus : C.I.I.,. X11I, 1×6.3, 2138. Patronus apiimus el pienlissimus : 1950. Patromus oplimus el indulyenlissimus: 1956 . I'alrona incomparabilis : 2296.

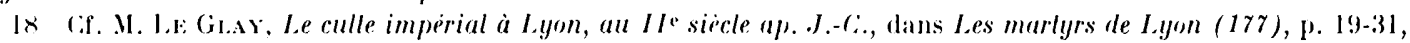
en particulier p. 2.4 et s. On a quelques raisons de localiser la schola des seniri Augnstales en haut de la montee de

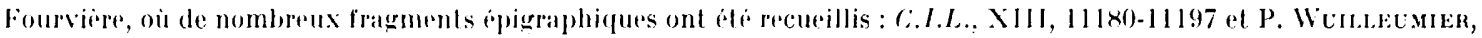
Inscriplions lalines des Trois fianles abrige : I.L.T.(i., Xlle Suppl. a liallia, 1963, 239-240. Sur les Augustales

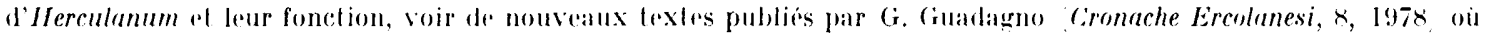
se trouse rassemble une bonne docmmentation. 
ronstate, en effet, que c'est all $\mathrm{II}^{\mathrm{e}}$ s. que l'institulion a atteint son apogée. Parmi les documents datables (ils sont all nombre de 17), un seul remonte à la première moitié du I $^{\text {er }}$ s.; trois seulement à la période 40-70; en revanche 38 sont datables entre 70 et 240 , la plupart de ces derniers appartenant plutôt au ${ }_{\text {II }}{ }^{\mathrm{e}}$ s. qu'aux premières décennies du $\operatorname{III}^{\mathrm{e}} \mathrm{s}$.

Ln autre intérêt du document lyonnais récemment exhumé est de le voir s'ajouter au petit nombre de ceux qui nomment des sévirs ayant exercé en mème temps une profession lucrative, et en particulier une profession lucrative organisée en association. Il n'est peutêtre pas inutile d'en dresser li liste :

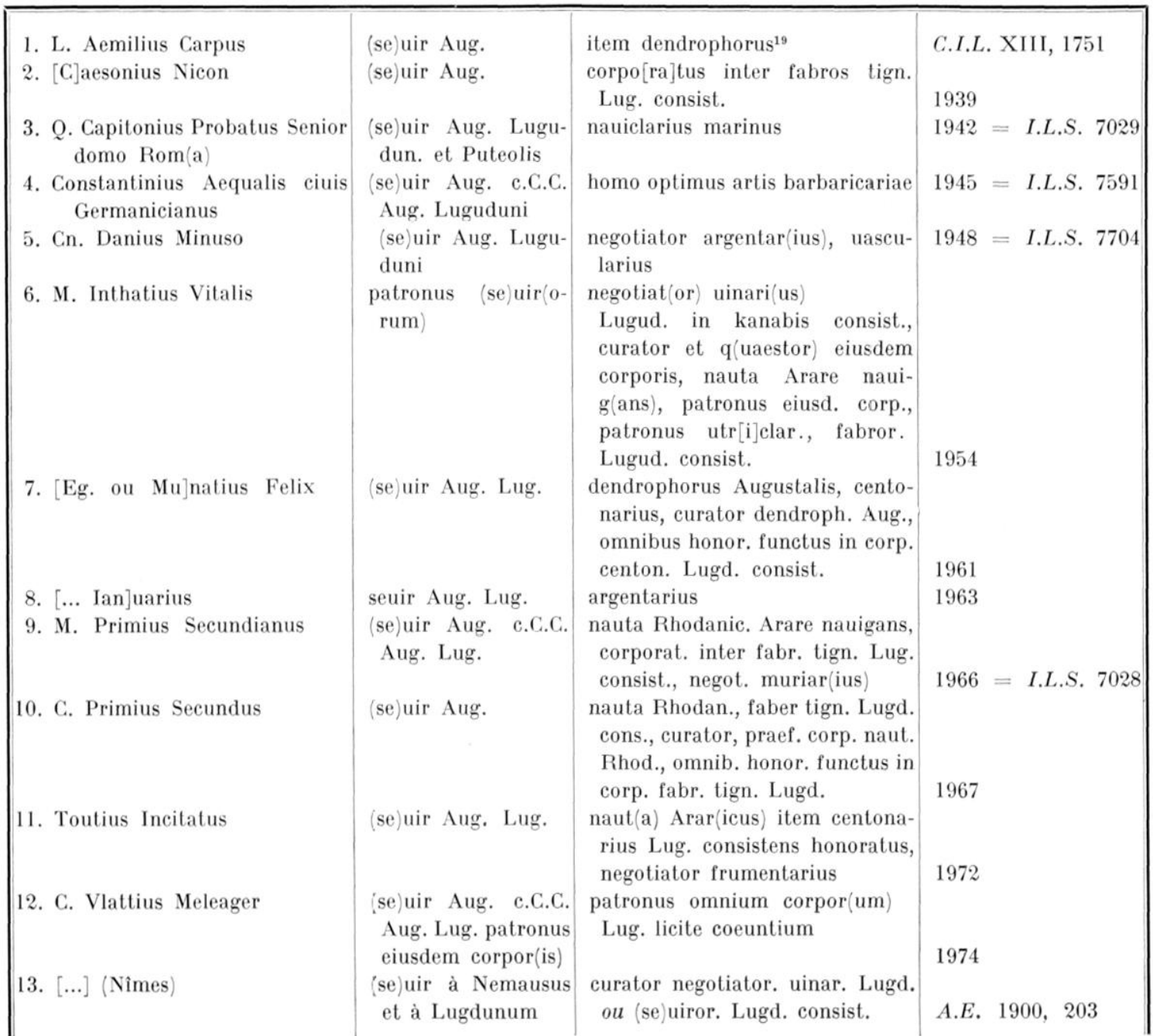

Sur les quelque 70 sévirs augustaux dénombrés, treize sculement et même onze, si l'on ne compte pas L. Acmilius Carpus dont la fonction de dendrophore n'est peut-être que religieuse et II. Inthatius Vitalis qui ne fut pas vraiment seuir Auguslalis, mais patron du collège - ont exercé une activité professionnelle, qui pour huit d'entre eux consistait

19 Dendrophortus na peut-être ici quiune signification religieuse, dautant qu'il s'agit d'une inscription

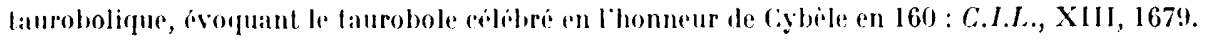


en une profession organisée en association. Mttalus vient dons s'ajouter à cette liste. Mais -- et ceci confirme encore une juste remarque de I. Carace Ruggini2o pas plus que la plupart de ses confrères dans le sévirat augustal, il n'a été jusqu'au patronat des corps professionnels auxquels il a appartenu : trois sévirs lyonnais seulement (les nos 7, 10 et 12) ont obtenu ce privilège. Attalus est palronus certes, mais il est le palronus oplimus incomparabilisque des quatre affranchis qui servent sa mémoire; il n'est pas le palronus d'une association professionnelle. Voilà qui confirmerait, s'il était nécessaire, la position sociale du sévirat, entre l'ordo decurionum et le populus ${ }^{21}$. Et qui montre bien qu'il n'y avait pas d'interdépendance, à Lyon, entre promotion sociale (au point de vie professionnel) et avancement politique ${ }^{22}$ d'une part et appartenance à cette "noblesse " d'origine affranchie - et largement gréco-orientale - - d'autre part.

Enfin, l'épitaphe d'Attalus permet de projeter une petite lumière supplémentaire sur une activité peu représentée dans la vie commerciale des Ciaules, le commerce des parfums. Dans la liste des métiers pratiqués à Lugdunum, qu'avait naguère dressée P. Wuilleumier ${ }^{23}$, on trouve, à côté de la sćrie imposante des professions organisées en corporations -- les plus nombreuses et les plus puissantes de l'empire, après celles de Rome et d'Ostic -. - un nombre important de métiers indépendants. Parmi ceux-ci on note un saponarius (C.I.L., XIII, 2030) et deux unguentarii (C.I.L., XIII, 2602; VI, 9998); Septimius Iulianus negolialor Lugdu[n(ensis) ar lis saponariae; Pisonius Asclepiodotus ung(u)entarius, seuir Aug. c. C.C. Lug. (texte de Chalon-sur-Saône = Cauillonum).

Il faut done distinguer le seplasiarius de l'unguentarius. Ce dernier ne serait-il pas plutôt un marchand d'unguentaria, c'est-à-dire de vases à parfums? Alors que le premier serait le véritable marchand de parfums? Cela expliquerait peut-être qu'alors qu'on ne connaît pas d'unguentarius dans l'armée romaine, une inscription de Mayence (Mogunliacum) mentionne un seplasiarius in leg(ione) I Ad(iulrice), sans doute fournisseur de parfums pour les thermes et pour les offrandes sacrificielles. Si l'on préfère conserver à unguenlarius son sens traditionnel de marchand d'onguents, le seplasiarius pourrait être un marchand de parfum d'un type particulier, le séplase (seplasium) qui se vendait sur une place de Ciapoue, à qui il avait donné son nom, la Seplasia, à moins que ce ne soit le contraire, c'est-à-dire que la place ait donné son nom au parfum spécial qui y attirait

20 Loc. cil., p. 74, 76-77.

21 Sur les générosilés financières des seuiri lyonnais, voir les exemples réunis par l. Cracco-Rcgani, loc. cil., p. 77 el note 1. Ils devaient acquitter une summa honoraria, à preuve l'inscriplion de Nìmes, qui nomme un seuir Augustalis de Lyon, Narbonne, Orange et Fréjus, dispense dans ces quatre villes de la s.h.: ubique graluitis honoribus (C.I.L., XII, $3203=$ I.L.S., 6984. Sur les dépenses des sévirs, cf. aussi R. Detroy, La fonclion sociale de l'auguslalité, dans Epigraphica 36, 197.4, p. 134-154 et P. Garssey, Descendants of Freedmen in local politics, dans B. Levick et al., The Ancient IItstorian and his materials: Essays in honour of C. E. Stevens, 1975, p. 167-180.

22 Mais non participation officielle à la vie civique, comme le rappelle G. Fabre, Libertus. I’alrons el affranchis à Rome, Rome, 1981, p. x. Voir toujours L. Cracco-RugGisi, loc. cil., p. 78.

23 Lyon, métropole des (iaules, 1953, p. 49-56. On releve a l yon quelque 40 inscriptions de métiers. A Narbonne, II. Gayraud, Narbonne antique des origines à la fin du $11 I^{\mathrm{e}}$ siecle, Paris, $19 \times 1$, relève 103 inscriptions faisant connaitre 105 Narbonnais exerçant un métier; il souligne les ressemblances ct. les différences entre les deux villes : des deux còtés prédominance du commerce sur l'artisanat; à Narbonne prépondérance des affranchis et des noms italiens et absence quasi totale des corporations. 


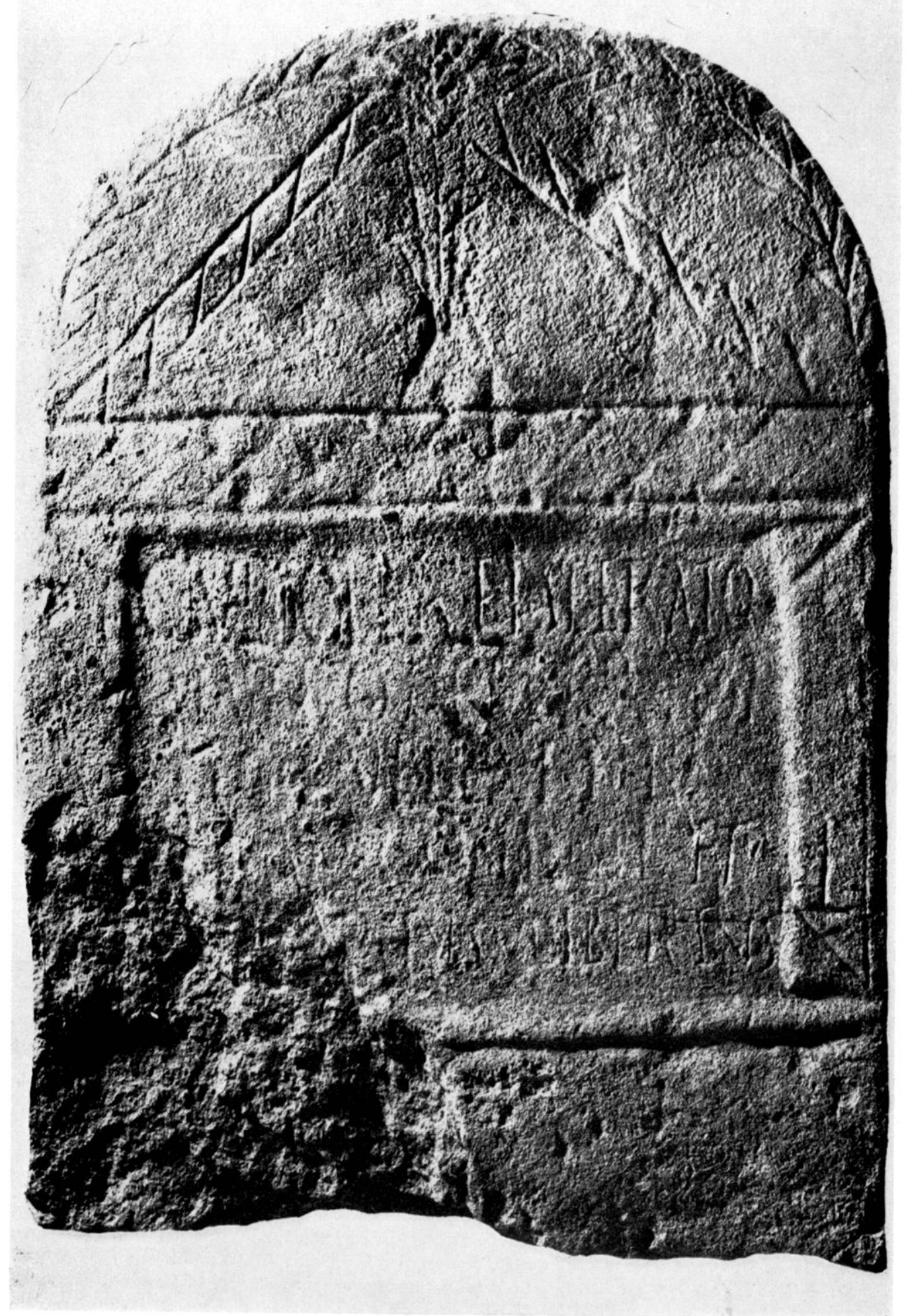

6 Ia slite de C. Iulius speralus. 
l'affluence ${ }^{24}$. La première explication me parail la plus vraisemblable. Peut-être arons-nous là une nouvelle attestation des relations commerciales nouses cnlre Lugdunum et la Campanie ${ }^{25}$. Pour terminer, j'ajouterai que du marchand de parfum (mercalor seplasiarius) dans l'exercice de ses fonctions, nous possédons une représentation sur un relief de Lillebonne, chez les Calètes (en Lyonnaise). Ce relief qui se trouve au musée de Rouen montre un personnage en tunique, debout derrière son comptoir. Il lève la main pour décrocher un outil pendu sous le dernier des trois rayons où sont déposés deux étages de boîtes de formes diverses. On a reconnu avec raison dans ces flacons de verre à col étroit et ces petites urnes à anse des vases à parfums et dans ce personnage "au profil sémitique» et aux "cheveux bouclés" un "marchand de vases et de verres" (R. Lantier), plus justement un "Syrien dans sa boutique de parfums» (P.-M. Duval) ${ }^{26}$. Attalus n'était probablement pas syrien, mais comme negotiator seplasiarius, il pouvait bien être quand même le fournisseur du marchand de Lillebonne.

2. Un cippe anonyme (fig. 5). La tombe T 91 était, elle aussi, constituée d'une cure de sarcophage monolithe, taillée dans la partie inscrite d'un cippe funéraire. Elle mesure 2,3う) $\mathrm{m}$ de long sur $0,72 \mathrm{~m}$ de large et 0,61 de haut. Mais, à la différence du document précédent, le tailleur de pierre a fait disparaître la quasi-totalité du texte gravé sur le cippe. Quelques lettres seulement subsistent sur les hords de la cuve. Peut-être la ligne 4 (si l'on ne compte que les lignes dont sont conservés quelques restes) portait-elle : $n$ [aula Ihodan. A]ra|[re nauigans] ? Ce n'est bien sùr qu'une hypothèse. La prudence commande plutôt l'abstention.

3. La stèle de C. Iulius Speratus (fig. 6). Dans une tombe était remployée une stèle de grès de $0,73 \mathrm{~m}$ de haut sur $0,38 \mathrm{~m}$ de large et $0,15 \mathrm{~m}$ d'épaisseur. Brisée en bas, elle porte un décor architectural gravé. Deux pilastres latéraux, qui délimitent le champ épigraphique $(0,29 \times 0,42 \mathrm{~m})$, supportent une architrave décoréc de croisillons et sommée d'un fronton triangulaire. En bordure du sommet arrondi sont gravées deux palmes. On peut lire, en lettres de 3,5 à $4,5 \mathrm{~cm}$ et non sans difficulté $\mathrm{s}^{27}$ :

24 Selon J. LE Gals, Métiers de femmes au Corpus Inscriplionum Latinarum, dans Mél. M. Murry, Revue des Etudes Lalines, 47, 1969, p. 124, l'unguentaria (esclave ou affranchic; est bien une marchande de parfums, ì distinguer évidemment de l'unctrix (C.I.L., XIV, 3035). Sur le seplasiarius et le seplasium, cf. Pi:Troxr, Sal., $76,6$. Sur la Seplasia de Capoue, dont parle notamment (ic., In Pis., 24 ; Sest., 19, voir bien sùr J. Hevrgox, Recherches sur l'hisloire, la religion el la civilisation de Capoue préromaine des origines à la deuxieme guerre punique, Paris, 19.l2, p. 122-123, 132-134, 439 .

25 Voir J. Rocgi, Aspects économiques du Lyon antique, dans Les martyrs de Lyon (177), p. 47-63, en particulier p. 5.3 ot $56-57$.

26 Sur le relief de Lillebonne (au musée des Antiquites de Rouen, no 17320;: Esprexavirc, IV, 3097; M. Hostovtzerr, Soc. and econ. Hist., p. 164 et pl. XXVIII, 2; R. I.avrıer, Ginide illustré Musée Sainl-Giermain, 1948 , p. 146 ; P.-.. Iuvar, La Vie quotidienne en Gaule..., 1952, p. 190 ; J. I)astidue et L. Harmaxi, dans M. DE Bö̈ARD, II isloire de Normandie, 1970 , p. 62 et pl. 4. Sur ce type de relief, voir L. M. BEssig.vict, La representation du travail dans la Gaule romaine these de 3 e cycle, Paris IV, 1974, ex. dact. et M. REDDE, Les scenes de métier dans la sculplure funéraire gallo-romaine, dans Gallia, 36, 1978, p. 43-63.

27 Plusieurs améliorations de la lecture initiale ont éte acquises lors d'un examen de la pierre en compagnic de II. J. Rougé, que je veux remercier ici. 


\section{(: IVIIO ILAE F SPERATO \\ SEVIRO (: (: LVG SIBI \\ E'T (C) VILLAE FII \\ i coxitrit ET \\ ATYS LIBERTIS}

Peut-ètre un I est-il gravé à la fin de la ligne 4, sur le pilastre de droite. On proposera de restituer : C(aio) Iulio Ilae f(ilio) Speralo | seniro c(oloniae) C(opiae) Lug(duni) sibi | et

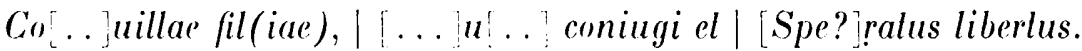

Si les tria nomina du défunt ne sont pas contestables, le nom de sa fille défunte n’est pas sùr, non plus que les noms de son épouse et de son affranchi qui ont érigé le monument. A la ligne 1, on lit, semble-t-il, sans grande peine le patronyme de C. Iulius Speratus, Ilae $f$. le nom apparaît pour la première fois à lyon, du moins sous cette forme, qui n'est sans doute qu'une variante, à peine latinisée. de IIylas (d'où Ilas) plusieurs fois attesté à Lyon mème. notamment pour les affranchis ${ }^{28}$. La mention du séviral seul, e'est-à-dire non accompagnée d'Augustalis est rare. Elle se rencontre toutefois d'abord sur le tombeau bien connu de Q. Caluius Q. I. Pal. Turpio, simplement qualifié de seuir (C.I.L., XIII, 1941), qui date du début de notre ère ${ }^{29}$ et sur deux inscriptions qui portent, l'une [se \}xuir Lagud. (C.I.L., XIII, 1813), l'autre (se)uir Lu[g.] (C.I.L., XIII, 1724). L'abréviation C.Ci.LVG pour déxigner la colonie de Lugdunum n'est pas non plus courante. Ces singularités, ajoutées à la nature de la stèle, à l'absence de I).MI. et de mention d'âge et à l'usage du datif pour désigner le défunt, inclinent à attribuer ce monument au $\mathrm{I}^{\mathrm{er}}$ s., au plus tard au début du II $^{\mathrm{e}}:{ }^{30}$.

4. Le cippe de Varia Reslitula (fig. 7). Bien que remployé dans une tombe, ce cippe n'a pas subi de grands dommages; seuls la base et le couronnement ont été abattus. Il mesure $2,11 \mathrm{~m}$ de haut sur $0,80 \mathrm{~m}$ de large et $0,76 \mathrm{~m}$ d'épaisseur. Très bien gravé en lettres de 3,2 à 7,5 con ; le texte se lit sans peine ${ }^{31}$ :

a) entre I) et $\mathrm{II}$ : manel hic | iunenis pia maler;

b) I)(is) M(anibus) | el memoriae La|riae Restilulae. | Haue mihi dul|cissimum nomen, | Varia Restilula, | coniunx mihi| quondam karis|sima meiq(ue) aman|lissima rari et mi|rae exempli inli|balae caslilis (sic) ab|slinentissimae | feminae cuius me|moria post obi|lum uiuel, uixil | an(nis) XXVI m(ensibus) V d(iebus) XX. | I'ale nimium cilo. T'(ilus) Valrius Myrismus mari|lus, (se)uir Aug(uslalis) Lug(uduni)| ol sibi uiuus fec(il).

28 Pour Hylas : C.l.L., XIII, 1970, 1997, 2007, 2156. Ylas : 2126 a Lyon mème et en Brelagne, à (Old Penrith (Voreda) : R.I.B., 9.37.

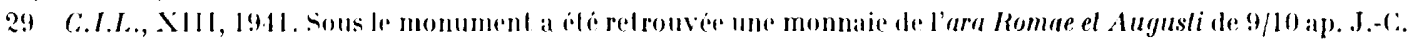

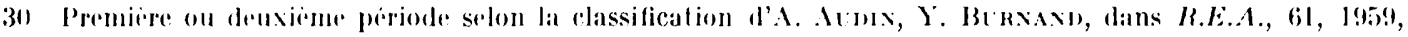
1). $321-322.2$.

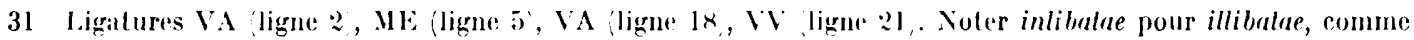
inlusler pour illusler : (..I.L., XII1, 5252, 5253, 7526. 


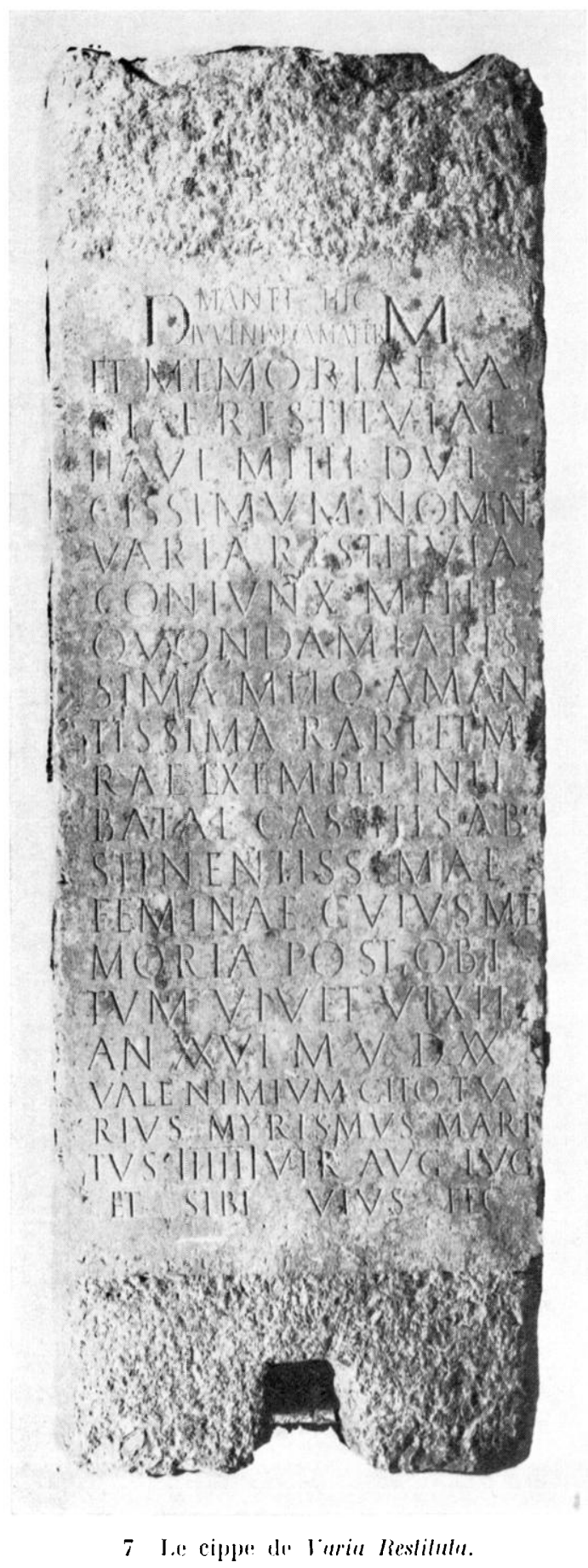

Il est bien possible que dans cette longue epitaphe de la inuenis pia maler Virria Restituta il y ait un effort de versilicalion, au vrai assez mal réussi ${ }^{32}$. Non sans grandes réserves, on peut en effet, de la ligne 4 à la ligne 18, décourrir un essai de système ïambo-trochaïque élémentaire, rombinant sénaires iambiques plus ou moins fautifs. septénaires trochäques à peu press corrects et un septénaire äambique correct (le sixième vers) :

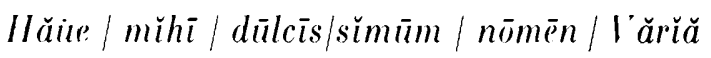

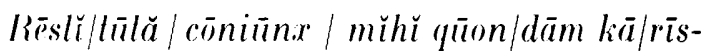
$\operatorname{simă~}$

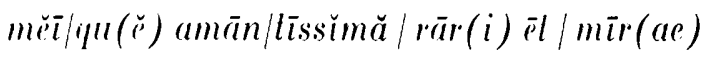
$\bar{e} x / \bar{e} m p l(i)$ in/lìbälläe

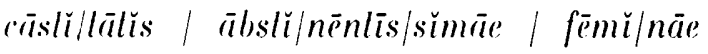
č̆lin̆s

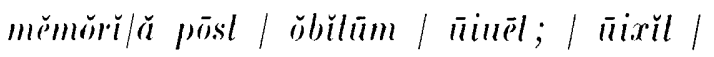
ännis

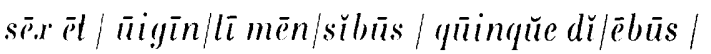
üiğin/lì

l'ălī / nimmàum / cùlōio.

En somme, le seul fait stylistique qui semble plaider en fareur d'une tentative de versificalion est lemploi de el dans rari el mirae exempli inlibalae casli(la)lis, emploi tout à fait anormal, avece une structure en chiasme entre arljectifs et substantifs. Et ce qu'il y a de mieux, n'est-ce pas la tripodie de conclusion? sauf que la répartition métrique concorde avec la division en mots ! Peut-être, après tout, faut-il croire simplement au hasard des combinaisons; et donc se garder d'interpreter ec lexte comme une intention poetique maladroitement exécutée?

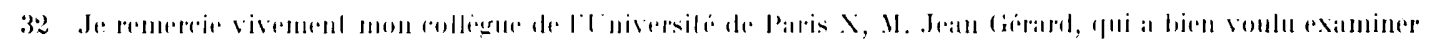

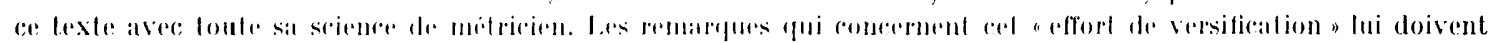

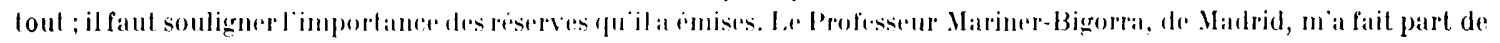
judicieuses observations, de ses reserves aussi. Qüil en soil remereir. On notera que lal qualité des épitaphes métriques trouvées a lyon est somvent tres mediocre el les essais de versification y sont presque toujours qualifies de médiocres :

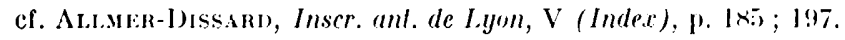


Ciela dit, remarquons que les Íarii ne sont pas très nombreux à Lyon, où ... en dehors du potier Sex. Varius ${ }^{33}$ - n'est guère connue qu'une Var(ia) Rufina, belle-mère d'un décurion ${ }^{34}$. Mais il faut noter qu'un L. Varius Lucanus se chargea du creusement d'un aqueduc souterrain à Voreppe, chez les Ambarres ${ }^{35}$. Cie qui suffit sans doute à classer cette famille parmi les notables aisés du pays. Varia Restituta, la jeune défunte de 26 ans et son mari éploré T. Varius . Yyrismus, nouveau sévir Augustal lyonnais, tous deux affranchis d'un même patron certainement ${ }^{36}$, devaient, à en juger par la qualité du monument et de l'épitaphe, participer à cette aisance et à cette notoriété.

Bien qu'elle s'insère dans une longue liste d'inscriptions lyonnaises célébrant non sans quelque emphase les qualités des défunts, -.. c'est le cas, semble-t-il, en particulier de celles des affranchis, sévirs ou non , celle que T. Varius Myrismus a fait graver à la mémoire de son épouse mérite de retenir l'attention.

Pour le ton, elle s'apparente à celle de Marcellina, fille de Solicia morte à 24 ans, dont Martinus, son mari célébrait la tendresse (carissima), l'âme très pure et du plus rare exemple (animae sanclissimae el rarissimi exempli) ${ }^{37}$. A celle aussi de la Cirecque de Nicomédie Tertinia Amabilis, morte subitement après dix-huit ans de mariage, pendant que son mari, Tertinius Gessius, vétéran de la 8e légion Auguste, était en voyage; elle était, nous dit colui-ci, une coniux karissima et pienlissima, caslissima conseruatrix mihi pientissima, fortuna praesens ${ }^{38}$. De plus près elle s'apparente également à l'épitaphe de Felicia Minna, femina rarissima, castitatis exempli adfectionis plenae erga omnes homines ${ }^{39}$. Et mieux encore à celle de I. Vindicia Luperca de Cologne, femina sanclissima summa castitale praedita ${ }^{40}$. Comme elles et même sur plusieurs points mieux qu'elles, Varia Restituta fut une épouse très tendre et très aimante, exemplaire, d'une chasteté parfaite (inlibatae castitatis), une femme tout à fait désintéressée (abstinentissimae feminae). Cette dernière vertu est en général plutôt attribuée aux hommes et surtout (!) aux administrateurs ${ }^{41}$. Aux femmes reviennent en revanche la tendresse, la fidélité, le zèle et la vertu exemplaire, la chasteté. Le modèle à ces égards fut sans doute Primitiuia Augustina, sanclissima femina quae uixit annis XXXXV cu[ius] fides castilas probitas diligentia obsequi immensa fuit numeralione $e^{42}$, un modèle dont Varia Restituta n'était guère éloignée.

Il y a pourtant dans son épitaphe des accents particuliers qu'on trouve moins fréquemment ou pour le moins diversement exprimés dans les inscriptions lyonnaises. D'abord,

33 A.L.MER-1)ISARD, op. cil., 19, p. 128.

34 C.I.L., XIII, 1924, ligne 14.

35) C.I.L., XIII, 2188 : aquam perluxit.

36 Myrismus est déja allesté a Lyon : C.I.L., XIII, 2:2xx. II est aussi possible que Varia Restituta ait été affranchie par son mari; vu son àge, cela me semble peru problable.

37 C.I.L., XIII, 2200.

38 C.I.L., XIII, 1897.

39 C.I.I., XIII, 1916.

40 C.I.I., XIII, 190\%.

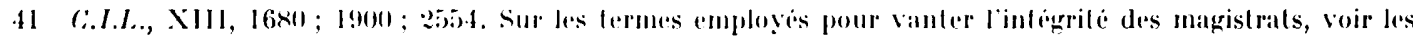

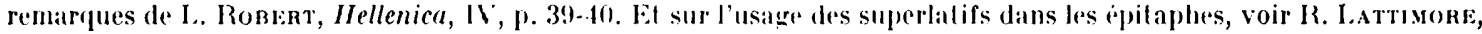
Themes in greek and latin Epilaphs, 1942, p. $290 \mathrm{et} \mathrm{s}$

42 C.I.I., XIII, 2244; cf. alussi 2237 , al I.L.T.(i., 250). 
dès le début du texte ${ }^{43}$, une sorte d'acte de foi dans la survie du souvenir de la défunte parmi les vivants, puisque, bien quappartenant désormais au monte des di Manes, elle demeure ici (manel hic), parmi les vivants, cette iuuenis pia maler... seule allusion du texte: à l'existence d'une descendance. Et cette confiance dans la survie de la memoria (qui n'es pas dite comme à l'accoutumée aelerna), elle réapparaît plus loin. fortement proclamée dans le cuius memoria post obilum uiue ${ }^{44}$.

Autre singularité qui dérive de la première : la répétition des appels directs à la défunte, plusieurs fois interpellée - - très logiquement d'ailleurs, puisqu'elle est toujours présente. C'est le sens, me semble-t-il, de IIaue, mihi dulcissimum nomen, Varia Restilula. Et l'adieu est repris à la fin, après la mention de l'âge: : uale nimium cilo $0^{45}$, qui fait écho ultime au iuuenis pia mater du début.

Tous ces traits particuliers, et significatifs, !’insistance mise à la fois sur la pureté et la chasteté de la jeune défunte, et sur la confiance dans la survie de son souvenir, ajoutés à l'abondance verbale dont témoigne le texte, et la nature même du monument - un cippe - permettent de le reporter au $\mathrm{III}^{\mathrm{e}} \mathrm{s}$. plutòt quau $\mathrm{II}^{\mathrm{e}} \mathrm{s} \mathrm{A}^{\mathbf{4 6}}$.

5. L'épilaphe de C. Claudius Florus (fig. 8). C'est une plaque de pierre à large bordure plate de $0,82 \mathrm{~m}$ de large sur $0,585 \mathrm{~m}$ de haut et $0,11 \mathrm{~m}$ d'épaisseur. Elle était remployè dans une tombe, comme les autres et sans retouches apparentes. Le champ épigraphique, en creux, mesure 0,405 sur $0,505 \mathrm{~m}$. Séparant les deux mots de la ligne 4 et les lignes 4 et 5 , deux palmes ont été gravées, symbole d'honneur et de victoire. Hauteur des lettres : $2, \bar{\partial}$ à $4,2 \mathrm{~cm}$.

C(aio) Cla(udio) Claudi(i) Maxi|mi f(ilio) Floro Sunuco, | Cllaudia) Senera maler f(aciendum) c(urauit); | ciuilas Aeduorum | l(ocum) s(epulturae) m(onumenlum ou -emoriam ou -onumenlique) p(osuil) d(edicauitque) ou p(ublice) d(onauil).

Bien que le texte ne contienne ni invocation aux dieux llânes, ni mention d'âge, ni formule de regret, et bien que la dernière ligne puisse ètre interprétée de plusieurs manières, il s'agit cependant, me semble-t-il, d'une épitaphe. La ligne う̃ pourrait par exemple être lue : l(ibens) $s$ (oluil) $m$ (erilo) $p$ (osuil) d(edicauilque), mais dans ce cas manquerait u(otum), le mot principal d'une formule qui ne convient bien qu'à une inscription en l'honneur d'une divinité. D'autre part, il faut remarquer que, si la formule abrégée l.s.m.p.d. n'est pas jusqu'ici attestée à $\mathrm{L}, y o n^{47}$, elle y est employée plus d'une fois disjointe

43 Pour une disposition du même type, voir C.I.L., X111, $1 \times 97$ : entre I) at ll est gravé Aue .1mabilis Gessio tuo karissi[m]a. Tertinia Amabilis estait l'épouse de Tertinius Gessius, véteran de la leg. VIII Aug., morte apres dix-huit années de mariage. Il y a cependant une différence avee le nouveau document lyonnais, où il ne s'agit pas d'une formule de salut. A noter aussi que l'expression hic iacet est parfois employée dans fe corps du texte; ainsi sur une stèle de Limoges : (:I.L., XIII, 1393. On trouve manel cin(is) hic à Autun : C.I.L., XIII, 2735.

44 Pour une formule encore plus élaboré, cf. C.I.L., XIII, $2077:$ memoria laudis eius el gloriae manenle hot titulo durabil aelerna. Faut-il rappeler le mot de Cicéron : Vita enim mortuorum in memoria est posita uiuorum (Phil., IX, 10)?

45 Pour des formules d'adieu du même genre, cf. C.I.L., XIII, 2058: Haue $\mid$ in aelernum $\mid$ Q. Anchari $\mid$

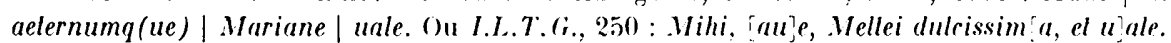

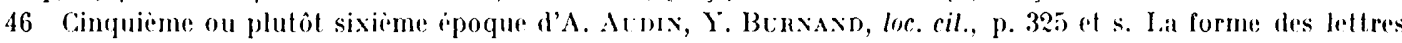
est, pour certaines d'entre elles, proche de C.I.I., XIII, 1679, date de 160. Mais la forme de cerlaines autres, telles que les $\mathrm{M}$, appartient plutòt au u $^{\mathrm{e}} \mathrm{s}$.

47 L (ocus) s(epullurae) à Narbonne: C.I.L., XI1, $4 \times 87$. 


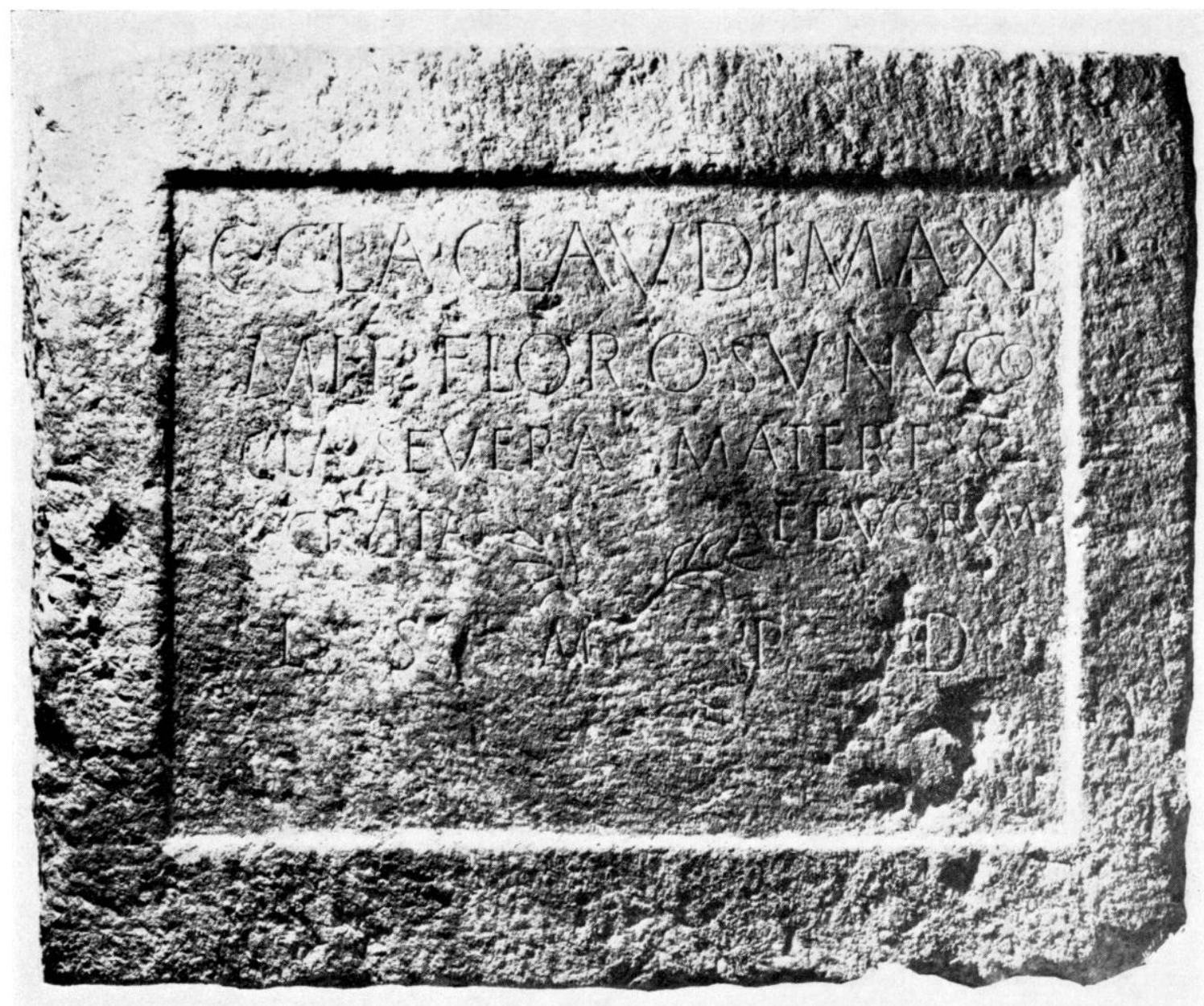

$\times$ L'epitaphe de C. Claudius Florus.

en deux parties. Memoriam posuil précedele el sub ascia dedicauil ${ }^{43}$ oul est utilisé sen ${ }^{49}$. Quant au locus sepullurae, il apparaît dans un rogglement du corpus fabrorum lign [uarior (um) (el) arlificum leclorum ${ }^{50}$, et il est offert par l'ord(o) sanclissim(us) de: Lugdunum a l'har(uspex) prim(us) X. Oppius Placidus ${ }^{51}$. On observera en outre qu'à Lyon on n'ignore: pas l'expression locus monumenti sepulluraeque donauil ${ }^{52}$. Pour ces raisons, C. Claudius Florus me paraît bien être un défunt dont la mère, Claudla Seuera a voulu honorer la mémoire et ériger la sépulture.

Les noms sont tres courants et il n'y a pas lieu d'y insister. En revandhe, plusieurs points méritent d'ètre soulignés. Aulant sinon plus que d: Claudlus filorus, son prere Cilaudius Maximus devait être un personnage d'un certain redief, d'où sans doute l'impor-

la (:I.I., XIII, J $\times 61 ; 2016$.

4! 6.J.L., XIII, 3406; 3157 ; 6:5ll.

(I) V.I.L., XIII, 1734.

51 C.I.I., XIII, 1721.

52 C.I.L., XIII, 2124. 
tance donnée au patronyme et l'intervention de la ciuilas Aeduorum, qui a tenu à fournir l'emplacement de la sépulture ${ }^{53}$. Il est d'ailleurs très possible que cette sépulture se trouve en réalité chez les Éduens, et qu'à Lugdunum n'ait été élevé qu'un cénotaphe. Cé qui expliquerait assez bien l'absence de référence aux dieux Mânes, de mention d'âge, de formule de regret et de dédicace sous l'ascia. De toute façon, la présence du monument à Lyon et les précisions que fournit son inscription à la fois sur l'origine du défunt et sur la ciuilas Aeduorum à laquelle il appartenait, ou à laquelle il avait de son vivant rendu des services qui lui valurent un tel témoignage de reconnaissance, viennent enrichir le dossier des relations entre la capitale fédérale et les peuples de Lyonnaise et de Belgique.

L'intervention d'une ciuitas en tant que telle n'est pas courante dans la capitale des Gaules. Elle est beaucoup plus naturelle au sanctuaire fédéral. Je ne trouve qu'un exemple, celui d'une dédicace faite par la ciuilas Lemouic(um) en l'honneur du sénateur lyonnais [...] Fidus A[...] Gallus Pacc[ianus ?] qui avait été gouverneur d'Aquitaine, dans la seconde moitié du $\mathrm{II}^{\mathrm{e}} \mathrm{s}$. probablement ${ }^{54}$; encore cetto dédicace des lémovices provient-elle sans doute de l'ara Romae et Augusli, c'est-à-dire du territoire fédéral plutôt que de la colonie. Si donc la ciuilas Aeduorum est ici mentionnèc pour la première fois, en revanche, les Eduens et surtout les Eduennes ne manquent pas dans l'épigraphie funéraire lyonnaise, toutes parées des plus belles vertus : Maria Macrina, Aedua, femina sanclissima, est pleurée à la fois par son mari Quintus Valerius 'Tertius, avec qui elle avait vécu 41 ans et 8 mois, et par son fils et sa belle-fille ${ }^{55}$; -.. Iulia Decmina, ciuis Aedua, femina oplima el sanclissima, est honorée par son héritier ${ }^{56}$; Tauria Titioli, ciuls Aedua, est célébrée comme une coniux carissima par son fils Maternus ${ }^{57}$.

Les Eduens ne sont pas tout à fait absents, si du moins l'on reconnaît l'un des leurs parmi les deux dédicants de la Fontaine au Cyclope érigée en l'honneur de Iupiler Optimus Maximus sur l'ordre de l'empereur Claude ${ }^{58}$. Il apparaît en somme qu'étaient oubliés, à l'époque de Claudius Maximus et de son fils C. Cilaudius Florus, les événements tragiques de l'année 68, qui avait vu les Eduens se regrouper avec les Séquanes, les Arvernes et les Viennois autour de Vindex, gouverneur de Lyonnaise, contre Néron, tandis que Lyon se tenait à l'écart, penchant plutôt du côté de l'armée de Germanie, appuyée par les Trévires et les Lingons. Les Éluens et les Arvernes avaient été écrasés par les cavaliers bataves. Ils n'en voulurent sans doute pas longtemps aux Lyonnais. Et les relations entre la capitale fédérale et le territoire arrosé par la saône ne pouvaient manquer de redevenir étroites, étant donné la communauté d’intérêts unissant les naulae Rhodanici et les naulae Ararici.

Il n'est pass moins intéressint de voir un Sunuque jourer un rôle chez les Éduens.

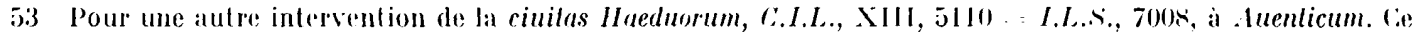
texle qui ne mentionne pas la colonie, mais la ciuilas Ileluel(iorum) date peut-être de l'epoque de (laude.

5) C.I.I.., XIII, 1803, qui date certainement dapres 138, puisque ce sénateur est sodalis IIadrianalis?.

5) $\quad$ :I.I.,., XIII, 8011 .

5f $1.1 \%, 1935,14=-J . L . T .1 ; .,: 353$.

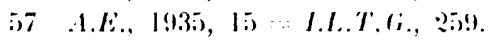

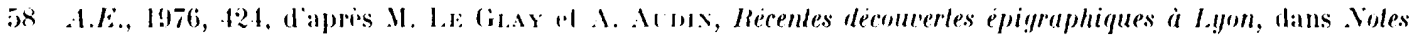
d'epigraphie el darcheologie lyonnaises, 1976, p. 6-21), en parliculior 1. 11. 
D'autant quil n'est pas le seul : à Beaune un autre Sunucus a recu de ses héritiers son monument funéraire ${ }^{59}$. Et à Lyon une Iu ... nia Clheunia (au surnom caractéristique), [domo S] inuco, a, en épouse très dévouée (coniux pienlissima), élevé un tombeau selon la coutume lyonnaise, elle l'a mème dédié sous l'ascia -- pour honorer la mémoire de son mari Quintinius Augustus, oplio de la XXIIe légion P'rimigenia P'ia Fidelis ${ }^{60}$.

Ces Sunici (ou Sinuci) étaient un peuple de la Gaule Belgique, voisin des Cerviens, qui en fait habitait la Germanie inférieure, entre la Veuse et la Roër61. Tacite, qui les appelle Sunici, leur fait jouer un rôle dans les troupes de Ciuilis en battant les anxiliaires romains sur la Meuse ${ }^{62}$. C'est done peut-être au cours des événements de 68-69 que les sunuques et les Éduens ont noué des relations dont la nature nous échappe malheureusement. Relations qui se prolongèrent jusqu'à Lyon. A cet égard, il est intéressant de voir la ciuilas Aeduorum honorer, dans la capitale fédérale des Gaules, un Sunucus de grand mérite, sans aucun doute.

La date de ces honneurs fait problème. Si l'on se fie à la chronologie habituelle des épitaphes lyonnaises, telle que l'ont établie A. Audin et Y. Burnand, l'usage du datif sans l'invocation aux dieux Yânes et la nature du monument - une simple plaque conduisent à la rapporter au $\mathrm{I}^{\mathrm{r}}$ s., avant 70. Pour des raisons historiques exposées plus haut, il me paraît difficile d'adopter une chronologie aussi haute. D'autre part, les caractères particuliers du texte, qui participe de l'épitaphe et de l'inseription honorifique, suffisent peut-être à justifier et l'absence des Di Manes et l'emploi du datif sur un monument qui me paraît appartenir plutôt à l'extrême fin du $\mathrm{I}^{\mathrm{e}} \mathrm{r}$ ou aux premières décennies du $\mathrm{u}^{\mathrm{e}}$ siècle.

6. L'épilaphe de Vera Exupera (fig. 9). Il s'agit d'un cippe réutilisé dans une tombe sans retouches apparentes. Il se présente sous la forme d'un autel funéraire sommé d'un fronton triangulaire, taillé en creux et dont le tympan est orné d'une ascia. Le fronton est flanqué d'acrotères pointus, portant les lettres D.I. Dimensions : $1.37 \mathrm{~m}$ de haut sur $0,71 \mathrm{~m}$ de large et $0,23 \mathrm{~m}$ d'épaisseur. Le texte de l'épitaphe est gravé sur la face principale de l'autel : champ épigraphique de 0,60 sur $0,63 \mathrm{~cm}$. Lettres de 3,6 à $1,1 \mathrm{~cm}$. On lit: $D$ (is) M(anibus) || et memori(a)e (a)elern(a)e| Forlianus Paler|nus coniugi cari|ssim(a)e Ver(a)e Exuper(a)e $\mid q u(a)$ e mecum uixil $\mid$ annis XXXIII m(ensibus) V d(irbus) XV $\mid$ p(onendum) c(urauil) (el) s(ub ascia) d(edicauil).

Notons que, dans tout le texte, les ae sont traités en e. Mais le phus remarqual,le est ici la dénomination par deux surnoms aussi bien pour la défuntr. Vera Lxupera ́pour Exsupera) que pour son mari Fortianus Paternus. Tandis que P'atemus est bien attesté à Lugdunum ${ }^{63}$. Fortianus y apparaît pour la première fois, comme il apparaît aussi,

5) I.I.L., XIII, 26.47.

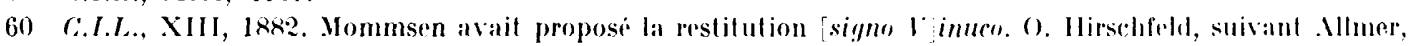
avait suggero Tnal. Sinuro. Ja leclure [S] inuro est dosormais assurie.

(i) Pi.I., N.II., IV, 17 .

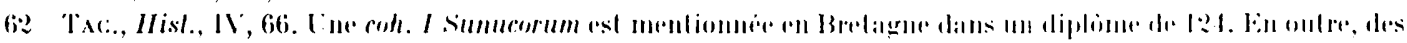

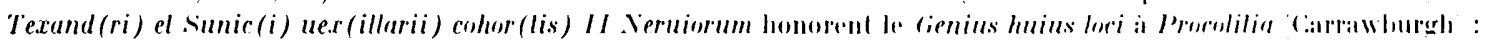
I.I..S., 2556 .

63 Par ix. C.I.L., Xill, 1845, 292x. 


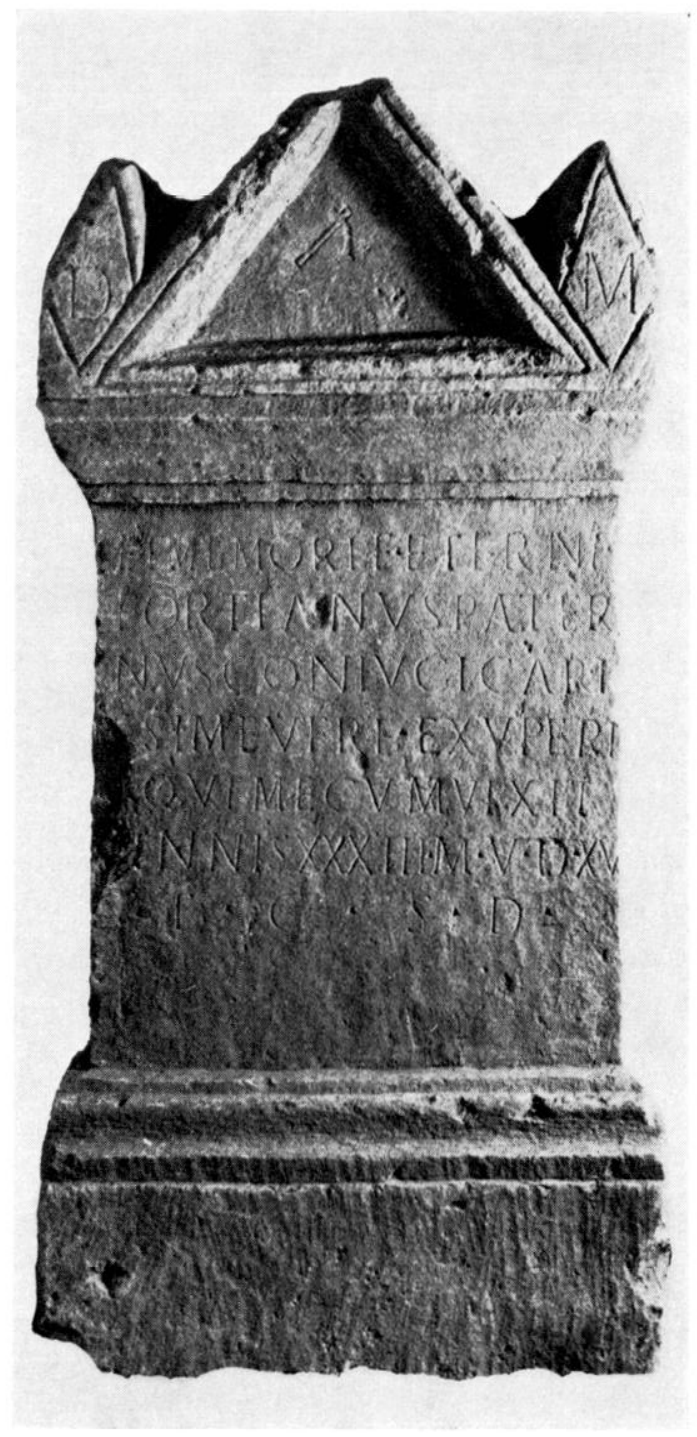

9) L'épitaph de Vera Eisnpera.

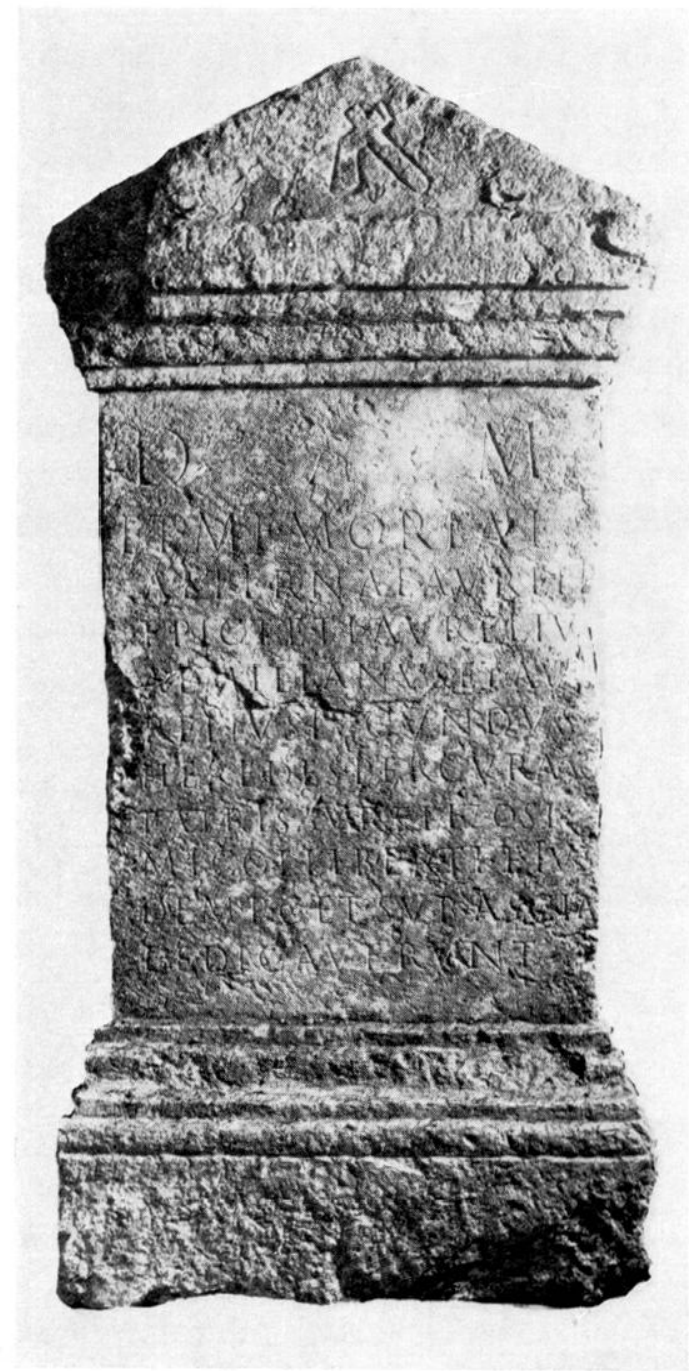

10 I'épitaphe d'Aurelius Épiclelus.

semble-t-il, pour la première fois dans les Trois Gaules ${ }^{64}$. C'est évidemment une forme dérivée de Fortis. De tels exemples d'allongement des noms abondent particulièrement à basse époque ${ }^{65}$; ils ne manquent cependant pas au $\mathrm{II}^{\mathrm{e}}-\mathrm{III}^{\mathrm{e}} \mathrm{s}$., période à laquelle appart ient l'épitaphe de Vera Exupera. Quant aux noms de cette défunte, le premier, Vera, est connu à Lyon ${ }^{66}$; Exupera en revanche ne l'était pas jusqu'ici67. Lil duplication du surnom sans

6.t Forlianus me tigure pas dans l'Index du C..I.L., NIII.

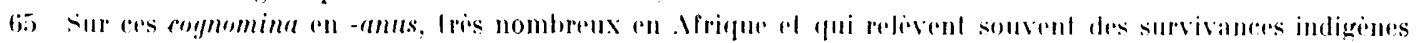

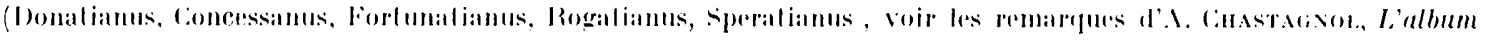
municipal de Timgad, 137s, p. 6010.

66 (:I.L., N1II, 190), I883.

67 Pour lixsupera, C.I.L., XII, 35); 


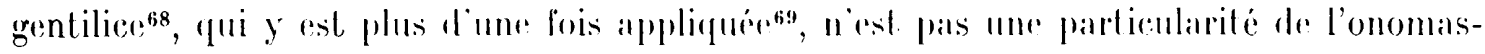
tique gallo romaine.

7. L'épitaphe d'Aurelius Epicletus (fig. 10). Elle est gravée sur un cippe, trouvé en remploi, mais qui a conservé sa base et son couronnement. Comme le précédent, il se présente sous la forme d'un autel funéraire à fronton triangulaire, mais celui-ci est lisse; il n'est ni creusé ni mouluré, mais simplement orné d'une ascia gravié. I)imensions : 1,04 m de haut sur $0,5 \mathrm{~m}$ de large et $0,45 \mathrm{~m}$ d'épaisseur. Le champ épigraphique courre toute la face principale, entre la base et le couronnement : $0,52 \times 0,45 \mathrm{~cm}$. On y lit en lettres de 2,1 à $4,9 \mathrm{~cm}: D$ (is) M(anibus)| el memoriae |aelernae Aureli(i)|Epicteti, Aureliu[s? | Aemilianus el Au|relius Iucundus | heredes per curam | palris Aureli(i) Zosi|mi colliberti eius|dem p(onendum) c(urauerunl) et sub ascia | dedicauerunl.

Comme le défunt, l'affranchi Aurelius Epictetus, tous les intervenants sont également des Aurelii, aussi bien ses deux héritiers, Aemilianus et Iucundus, que leur père Aurelius Zosimus, colliberlus du défunt. Sans doute les deux héritiers ćtaicnt-ils trop jeunes au moment du décès d'Epictetus pour s'occuper eux-mèmes de sa sépulture, ce qui expliquerait Ie per curam palris. Cees quatre Aurelii viennent s'ajouter à la longue liste des Aurelii lyonnais - - une trentaine - - parmi lesquels abondent les affranchis. Dans cette liste, où les noms hellénisants ne manquent pas (Cialocaerus, Demostenes, Trophimus, (aillistè, etc.), Eictetus et Zosimus prennent leur place ${ }^{70}$ à côté des surnoms plus latins Aemilianus ol Iucundus. Comme l'épitaphe précédente, celle-ci doit dater de la fin du Ir $^{\mathrm{e}}$ ou de la première moitié dı III ${ }^{\mathrm{e}}$ siècle.

II. I.

\section{Ia xécropolfe de ta RUE PIERRE-AUdry}

\section{A. Les déconverles archénlogiques.}

Faisant suite à la nécropole de Trion ${ }^{71}$ un ensemble de sépultures avait déjà été retrouvé dans cette zone, située en contrebas de la colline de Fourvière. En 1973 notamment avaient été fouillés des enclos funéraires ${ }^{72}$. En 1980, une fouille de sauvetage a permis d'étudier deux nouveaux enclos, disposés perpendiculairement à la pente de la colline. Ils reposaient sur un remblai de loess, de galets et de schistes. Chacun d'eux forme un carre de $4 \mathrm{~m}$ de côté. Sur les fondations en galets liés à l'argile, les murs sont construits, pour les premières assises, en bloes de schiste disposés en arêtes de poisson, pour les assises supérieures, en

6r Chacun sait qu’a Rome, les cognomina multiples apres un gentilice sont fréquents dans lonomastique des

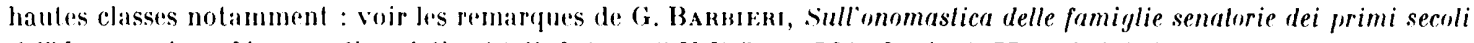

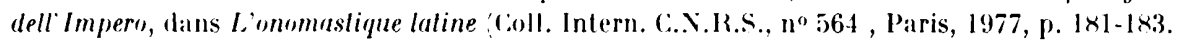

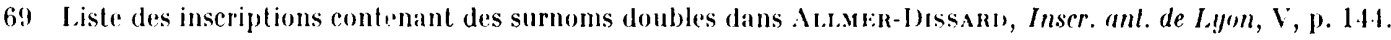

70 Sur Epictetus, cf. C.I.L., XIII, 1799 ef supra, p. 130. Sur Zosimus, ef. par (x. C.I.L., XIII, 217.4, 2235.

7) A. Atus, Topographie de Lugdunum, Jyon, 1953, p. 119 et s.

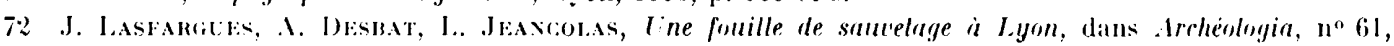
aouit 1973, p. $72-73$. 


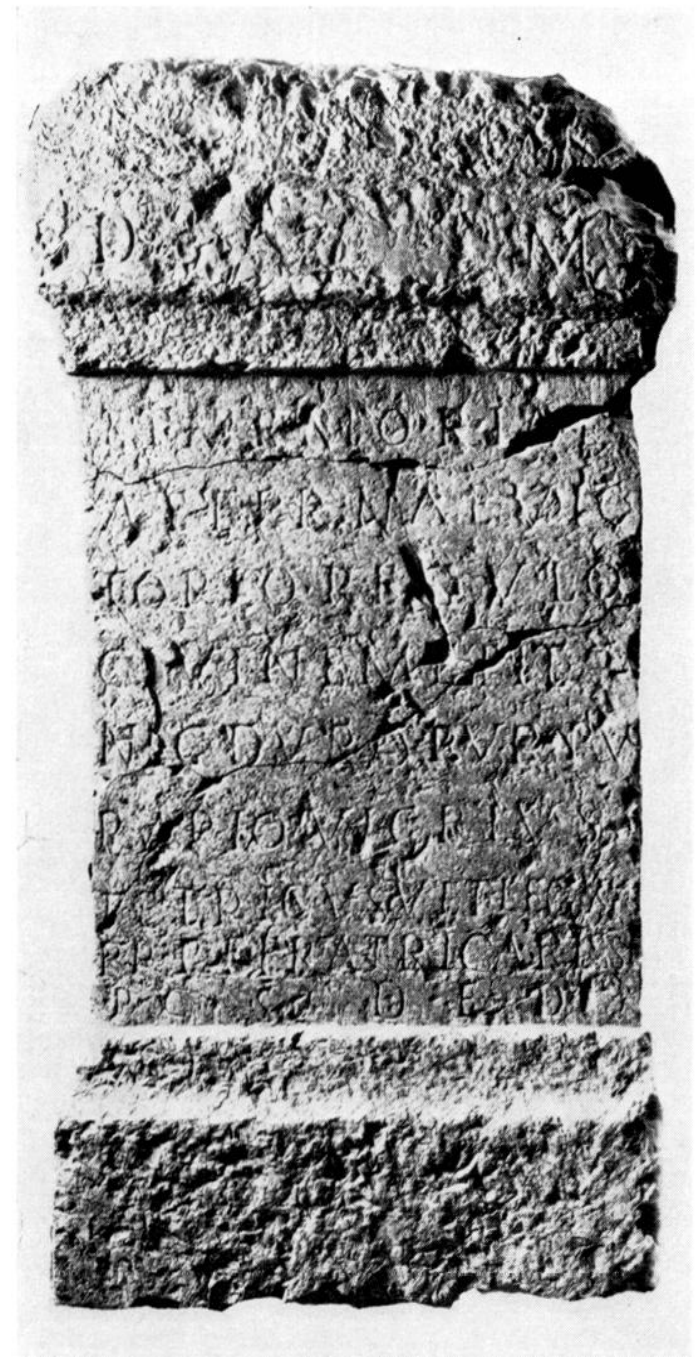

1I Jo rippe de Virlorius Regulns.

pierres vallaters régulièrement assemblées. Parmi celless-ei apparaissent des fragments d'arehitecture remployés.

L'un des enclos conservait encore en son eentre une stèle funéraire inscrite. Brisée en plusieurs morceaux, jointifs, elle grisait sur le sol en terre battue, au milieu d'une et probablement deux rouches de cendres et de charbon de bois, indiquant l'existence de deux foyers successifs. La stèle retrouvée doit correspondre au dernier foyer d'incinération.

Pour ces deux monuments funéraires les éléments de datation sont infimes. seule la présence de tessons de sigillée claire B dans le remblai antérieur à leur installation permet d'affirmer que la nécropole a dù s'étendre à ce secteur dans le rourant dlu rie siècle.

B. H.

13. Le cippe de Viclorius Regulus (fig. 11). En forme d'autel funéraire, ce cippe, avec sa base et son rouronnement, mesure $0.63 \mathrm{~m}$ de hatut sur $0,35 \mathrm{~m}$ de large et $0,21 \mathrm{~m}$ d'épaisseur. Hormis la mention des dieux Mànes, gravée sur le bandeau supérieur, le champ épigraphique occupe toute la face principale comprise entre les moulures plates de la base et du couronnement, soit toute la surface du dé, $0,33 \mathrm{~m} \times 0,30 \mathrm{~m}$. Les lettres mesurent de $1,6 \mathrm{~cm}$ à $2 \mathrm{~cm}$.

On lit: I)(is) M(anibus) || el memoriae | aeternae l"ic|lorio Regulo | ciai Nemeli el | neg(olialori) Duro(cortoro) purpu|rario, Vicrius (sic) | Telricus uel(eranus) leg(ionis) $X X[I I] \mid \operatorname{Pr}$ (imigeniae) $p$ (iae) f(idelis) fratri cariss(imo) $\mid p($ onendum) $c($ urauil), $s(u b)$ (ascia) ded(icauil).

Le texte pose d'abord un petit problème d'onomastique. Alor's que le défunt s'appelle Victorius Regulus, son frère, auteur du monument funéraire, est curieusement dénommé Vicrius Tetricus. Xon seulement les deux gentilices diffèrent - ce qui, en soi, ne crée pas une difficulté insurmontable, il pourrait s'agir par exemple de demi-frères ${ }^{73} \ldots$, , mais surtout

73 Les exemples de freres de noms différents ne manquent pas. Ainsi I. Silanius Candidus, M. Silanius Sabinus el T. Siluius Similis : C.I.I.., XIII, 5056. Ou C.I.L., XIII, 688I qui nomme Victorius (aissianus, paler avec ses filii Victorius Clementinus, Victorius Victorinus et Senecionius Iulianus. Ou encore C:I.L., XIJ, 8790, où sont mentionnés comme fralres I. Iustius Satto at L. Secundinius .Ioderatus. 
Vicrius est absolument inconnu comme nom ${ }^{73}$. Il faut done supposer a la ligne 7 une faute de gravure de la part, d'un lapicide distrait qui a dì omettre une syllabe75, et il convient donc de lire Vic(to)rius. W'autant que ce gentilice, quelque peu triomphaliste, est, tout comme les cognomina des deux frères ${ }^{76}$, attesté à Lyon et extrêmement répandu chez les peuples rhénans d'où est originaire Victorius Regulus ${ }^{77}$.

C'est en effet un des intérêts principaux de ce nouveau texte de nous révéler pour la première fois un Némète dans la population lyonnaise. Les inscriptions y araient déjà fait connaitre de nombreux représentants des peuples de Gaule Belgique et des deux Germanies ${ }^{78}$. Par ordre d'importance numérique décroissante, on peut citer, pour les civils :

1) des Trévires:

C. Apronius Raptor, Treuer, dec(urio) eiusd(em ciuitatis, n(aula) Araricus, palromus eiusdem corporis, palronus des neg(otiatores) uinarii (C.I.L.. XIII, 1911,.

$\mathrm{Cu}[$ pi]tus Ingenui f. ou $\mathrm{G}$. Vi _.. ] jus Ingen[uus], cinis Treuer (C.I.L., XIII, 1977).

Cacuronius Satto, Treuer (C.I.L., XIII, 1984).

T. Veratius Taurus, nal(ione) Trener (C.I.L., XIII, 1988).

C. Mansuetius Brasus, ciuis Treuer (C.I.L., XIII, 2012).

L. Secundius Octauus, Treuer (C.I.L., XIII, 2027).

M. Sennius Metilis, Treuer, negolialor corporis splendidissimi Cisalpinorum et Transalpinorum, praef(ectus) de ce mème corpus, faber tignuarius Lag(uduni) (C.I.L... XIII, 2029).

Terminalis, Treuer (C.I.L., XIII, 2032).

[...] ranius $\mathrm{V}[\ldots]$, ciuis Treuer, negoliator uinarius et artis crelariae Lug(uduno) consistens (C.I.L., XIII, 2033).

M. Tertinnius Katus, Treuer (C.I.L., XIII, 11200), ou Yatus (selon J. Kirier, I) ie Treverer ausserhalb ihrer Cinilas, Trier, 1981, p. 28 et s.).

2) des Séquanes :

Pompeius Catussa, ciues Sequanus, tector (C.I.L., XIII, 198:3).

Decimia Decmilla, ciuis Seq(uana) (C.I.L., XIII, 1990).

Diuixtus, ciuis [Seq]uanus (C.I.L., XIII, 1991).

Poppilius, natio[ne? Sequanus, negolialor arlis prassariae. honoralus corporis ulriclariorum (C.I.L., XIII, 2023).

3) des gens de Cologne :

Varenius Lupus, ciuis Agrip(p)inen(sis) (C.I.L., XIII, 2037).

Victoria Vrsula, ciuis Agrippinens(is) (C.I.L., XIII, 1904)79.

L. Vindicia Luperca, ciuis Agrippinensis (C.I.L., XIII, 1905) ${ }^{80}$.

74 Virius existe comme gentilice, mais non Vicrius : cf. C. Virius Victor at I. Virius Vitalis a Vienne : C.I.L., XII, 1809. Un exemple cependant à Bou . Xjem (Golas) : A.E., 1972, 681.

75 Il est fré(quent qu une lettre soit omise ; ainsi Resli(l)u lu]s dans C.I.L., XIII, 12048, ou inco(m)parabili (8072, 8377), ou Alexa(n)dri el faciu(n)dum $(3990$, secu(n)dus 6911, Pom(p)lina 1768 , Augu(s)la 6870 , etc. L'omission de deux lettres est beaucoup plus rare : voir plus haul castilis pour casti(la)lis, p. 135.

76 Victorius à Lyon : C.I.L., X111, 2039, 2057, 2117, 224x, ctc. Regulus : C.I.L., XI1I, 1986, 2584, etc. Tetricus : C.I.L., XIII, 2993, etc.

77 Victorius à Moguntiacum : C.I.L., XIII, 6681, 12;6881;6705 = 11810 , à titre d'exemples simplement. Regulus et Tetricus : 8094 bis ; 5010 , etc.

78 Des peuples et des villes-colonies ; on ne peut évidemment pas, sans précaution, mêler un séquane comme I)iuixtus et les ciues Agrippinenses de Cologne ou les Traianenses de Xanten.

79 Épouse d'un miles leg. $X X X$, Titiconius Verinus.

80 Épouse d'un b(ene)f(iciarius) proc., Iul(ius) Superinius Victor. 
1) des gens de Xinlen :

1. Sept imius Peregrinus Mdelfus, Traianens is (C.I.L., XIII, 1892)

Valerius IIonoratus, natione Troianensis (C.I.L., XIII, 20:34).

Adiuloria Perpetua, ciuis Traianensis (C.I.L.., XIII, 1976).

5) des I.ingons :

T. Tinc(ius', Mpinus, dec(urio) Lingon(um), I Iuir (C.I.L., XIII, 1922).

Valerius Sattiolus, ciu is Li]ng!o]nensis, negolialor arlis raliariae (C.I.L.. XIII, 2035)).

(6) des Rimes:

C. Iatinius Reginus, Remus. sagar(ius) Lugud. (C.I.L., XIIT, 200)8).

Nelianus, cines Remus (C.I.L.. XIII, 1796).

7) un Triboque :

II. Mtlonius Restilutus, cinis Tribocus, negolialor arlis macellariae (C.I.L., XIII, 2018).

8) un Vangion:

C. Nouellius Ianuarius, ciuis I Ingio, naula Araricus, curalor el palronus eiusdem corporis (C:I.L., XIII, 2020).

1 qui s'ajoutent, parmi les mililaires, retraités en général, morts à Lyon :

II. Altius II.f. Cla. Varcel lus . Ira Agripp(ina), miles coh(ortis) XIII urb(anae) (C.I.L... XIII, $18: 36 j$.

Morlestinius Peregrinus. ciu(is) Igripp(inensis), nel(eramus) leg(ionis) I M(ineruiae) (C.I.L., XIII. 1841

II. Aurelius) Primus, ciuis Rem(us), ueteranus leg(ionis) I Mineruiae (C.I.L., XIII, 1844). Quintinius Primanus, cinis Trener, nel(eranus) ex leg(ione) $X X X V \operatorname{lp}($ ia) Viclr(ice) Alexandrian(a) (C.I.L... XIII, 188:3).

Celerinius Fide lis, cinis Balanns, mil(es) leg(ionis) XXX (C.I.L., XIII, 1847).

El parmi les femmes de soldats :

Murastuia Fortunata, ex Ger(mania) Sup(eriore) ${ }^{82}$, cpouse de seplimius sextianus, mil(es) leg(ionis) VIII lug(uslae) (C.I.L., XIII, 1874).

Comme on voit, auceun Vémete jusqu'à notre Victorius Regulus, n’était jusqu'ici atteste a I yon, alors que les peuples voisins, Triboque et Vangion, l'étaient. Chacun d'eux l'est maintenant par un représentant. Et ainsi gràce au nouveau document épigraphique. se trouve reconstitué a Lyon ce triptyque de peuples frères qu'évoque, unanime, la tradition de César ${ }^{83}$ à Tacite ${ }^{84}$ en passant par Pline l'Ancien ${ }^{85}$. Tacite $(A n n ., 12,27)$ précise que sous

81 Il pourrait siagir d'Augusla Traiana an Thrace, le pere de I. Septimius) Peregrinus, I. Septimius Mucianus, m(issus) h(onesta) m(issione) ex leg(ione) XXX V.V.p.f. etant domo Philippopoli Plovdivi : C.I.L.,

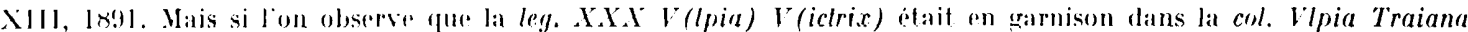
Santen - Veleri, on pensera plutot a cette derniere ville comme origrine de ce Traianensis et des deux suivants.

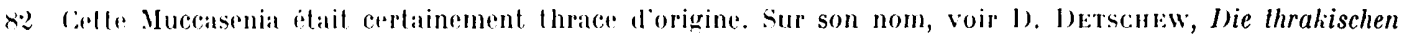

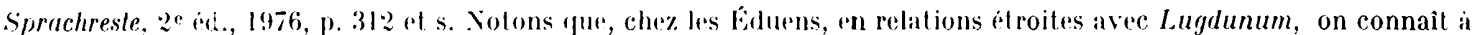
Cauillonum 'chalon-sur-sane, un veteran de la leg. XXX V.V., lustinius Mercator, cinis Treuer et sa femme, Natiuia Valentina, cinis Agrip(p)inensis : C.I.L., XIII, 261.1; w un Rime, eques alae Longinianae en garnison a Bonn sous

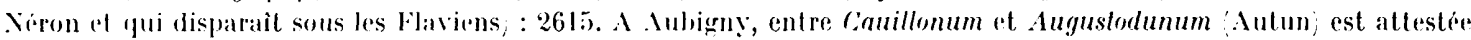
une Tertinia Fopentinia, ciuis Sueba Vicres: C.I.I., XIII, 2633. EL a Augustodunum on trouve un ciuis Treuer, senir Hug. in Leduis consistens, omnibus honoribus inler eos funclus :2669 al un medicus .Mediomatrix: 2674;.

×3 Cals., b. G., I, jl ot VI, :jo.

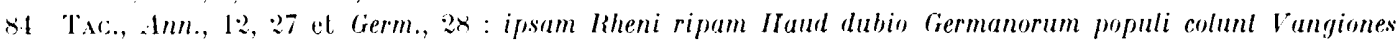
Triboci vemeles.

85) Pr.x, ․II., 4, Inf: Rhenum ... acculentes Germaniae genlium in eadem prouincia Nemeles Tribuci Vangiones. 
Claude les Yémètes al les Vangions fournirent de nombreux auxiliaires à l'armée de Germanie. Plus tard, on trouve des Némètes non seulement dans les ailes ${ }^{86}$, mais dans les légions et tout particulièrement dans la legio XXII Primigenia pia fidelis, stationnée à Mogunliacum (Mayence). C'est à cette légion qua appartenu, comme centurion, le Nemes L. (iellius I. f. Flauia Celerianus, qui en 204 fit à l)iane Auguste une dédicace pro salute des Augustes Septime Sévère et Caracalla et du César Ciéta ${ }^{87}$. Certes le nouveau texte lyonnais ne présente pas Victorius Regulus, cinis Nemes, comme un militaire, mais son frère, lui, avait servi dans cette mème légion qui, avec la I Mineruia, la $X X X$ Vlpia Victrix et la l'III Augusla, envoyait depuis 197 des détachements à Lagdunum pour y remplacer la XIIJe cohorte urbaine, dissoute après la défaite de Clodius Albinus ${ }^{88}$. Vic(to)rius Tetricus vient ainsi compléter la liste déjà assez longue des soldats et des vétérans de cette unité légionnaire, installés et morts dans la capitale des Gaules.

Soldats et vétérans de la legio XXII Irimigenia morts à L,con :

Albanius Potens, uet. (C.I.L., XIII, 1830).

Attonius Constans, uel. (C.I.L., XIII, 1837).

Augustius Augustalis, nel. (C.I.L., XIII, 18:38).

Cornelius Victor, uet. (C.I.L., XIII, 1851).

C. Iul(ius) Placidinus, uet. (C.I.L.. XIII. 186:3).

G. Mansuetius Tertius, uel. (C.L.L., XIII, 1868).

Cauonius Belli ${ }_{\mathrm{t}} \mathrm{c}$ jus, miles (C.I.L., XIII, 1872).

Peruincius P lacidus, uet. (C.I.L., XIII, 1877).

Quintinius Augustus, optio (C.I.L., XIII, 1882).

C. Verecundius Verinus, uel. (C.I.L., XIII, 19(2)).

Vrogenius Ners ou Nertes, uet. (C.I.L.. XIII, 19(7)).

Ce qui frappe dans cette liste, e'est quaucun de ces vétérans, non plus que ceux des autres légions d'ailleurs, n'a joute un rôle dans la vie municipale lyonnaise, alors que les militaires issus des deux cohortes urbaines qui tinrent garnison à Lagdunum jusqu'en 197, y avaient, quant à eux, rempli des fonctions municipales importantes ${ }^{89}$. Il n'est pas moins surprenant de ne leur voir jouer non plus aucun ròle dans la vic économique. A l'exception d'un seul, il est vrai, un vétéran de la legio I Mineruia, Vitalinius Felix, qui fut negolia[lo]r Lugdunensis artis cr e e]lariae ${ }^{90}$. Hormis ce marchand de poterie, aucun ancien militaire n'a exercé soit un métier indépendant, soit une profession organisée en corporation.

En revanche, notre Némète Victorius Regulus a été - mais c'était apparemment un civil - neg(olialor) purpurarius à Durocorlorum, c'est-à-dire à Reims. Mention

\footnotetext{
Ies Triboques avaient pour chef-lieu Vrocomagus Brumath, les Vingions Borbelomagus Vangionum (Worms) et les

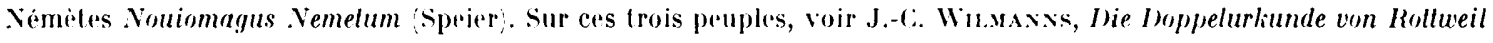
und ihr Beilrag zum slädlenesen in Oberyermanien, dans Epigr. Sludien, 12, 1981, p. 1-182, en particulier p. 96 et s. A noter que, si te gentilice Victorius n'est pas alteste jusqüici che\% les Németes, on y troure les surnoms Victor '(AI.I., XIII, 6096, Viclorinus 6076,6093 .

x6 Ainsi Niger Aetonis f. Nemes dans l'ala Pomponiani à Bonn : C.I.L., XIII, 8097.

xi C.I.L., XIII, 6659) I.I..S., t.2x, a Seligenstadt ad Moenum.

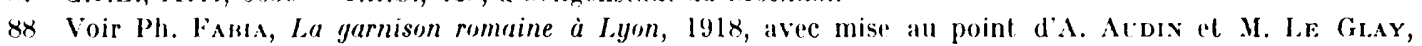
Nouvelles inscriplions de Lyon, dans . Voles dépigraphie el d'archéologie lyonnaises, 1976, p. $4 \times$.

89 Ibid., p. 47-51.

90) C.I.I., XIII, 1!)66.
} 


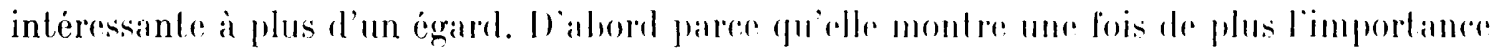

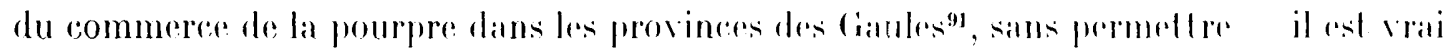
de préciser s’il s'agissait de pourpre importé de Phénicie al de Tyr en particulier, on de cet «ersatz» extrait d'herbes dont Pline fait honneur aux Gaulois transalpins ${ }^{92}$. Qu une telle activité ait été exercée à Durocortorum n'a rien d'étonnant ; on sait que le textile était une, des activités des Rèmes ${ }^{93}$. Une activité qui les mettait en relation commerciale aver Lyon, où un autre Rème, quant à lui, installé dans la ville, C. Latinius Reginus, sagar(ius) Lugd(unensis), y était mort lui aussi94. Io nouveau texte confirme ces relations, mais en insistant bien sur le fait que Victorius Regulus ne résidait pas dans la capitale fédérale, qu'il avait bien Durocorlorum comme siège de son activité et que, s'il est mort à Lyon, c'est qu'il y séjournait en "étranger", pour ses affaires très probablement ${ }^{95}$. C'est pourquoi, contrairement à l'habitude, on a bien précisé ciuis :Vemeli el neg(olialori) Duro(cortoro) purpurario ${ }^{96}$. Enfin, il apparaît bien qu'en Gaule, comme en Phénicie, les purpurarii appartenaient - la qualité de la sépulture, le formulaire de l'inscription et la référence au vétéran légionnaire le montrent -- a la classe aisée des semi-notalles locaux ${ }^{97}$.

Cés huit textes, on l'a vu, sont d'un intérêt inégal. Tous apportent quand même leur lot d'informations et viennent enrichir notre connaissance de la vie économique et sociale dans la capitale des Trois Gaules. Iien privilégié entre le monde méditerranéen et les régions frontières du Nord, Lugdunum, cité cosmopolite, industrieuse et dévote, méritaitelle dès l'Antiquité le jugement de Baudelaire, qui la qualifia, un jour, de "ville singulière, bigote et marchande»? Le rapprochement est sans doute force et trop facile. Il reste qu'à Lyon, comme à Vienne, mais sous des formes différentes et malgré une romanisation profonde, s'affirment des originalités qui restent à itudier, notamment en ee qui concerne la vie artisanale et commerciale98.

II. I.

\section{Jean-François Rlyxaud, Benoit Heiry et Marcel La (iLAY}

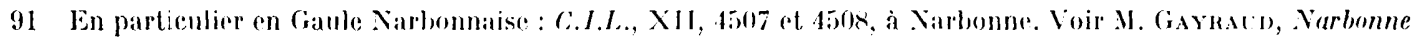
anlique des origines à la fin du $11 I^{\mathrm{e}}$ sibcle, 1981, p. 490. Aucun purpurtarius ne fiqure dans les Indices du C.I.L., XIII.

92 PuI., X.II., XXIII, 3 : les Gaulois Tramsalpins reproduisent avec des herbes la pourpre tyrienue, re qui leur évite d'aller chercher le murex au fond des mers. On sait que lat production de la pourpre, spécialite de Tyr, est attestéc aussi à Délos : cf. Ph. Broxwat, dans Bulletin de Correspondance IIellénique, 93, 1969, p. 759-791 ; 102, 1978 ,

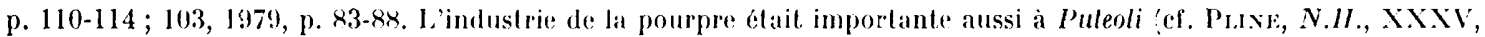
44-45; I.It., I, $\left.1(1981), \mathrm{n}^{0} 26^{*}\right)$ et a Tarente: voir J.-P. Mores, art. cil., infra note 98;.

93 C. a IJurocortorum l'épitaphe d'un uestiarius : C.I.L., XIII, 3263; of a Lyon, l'ipitaphe de C. Latinius Reginus Remus sagar(ius) : 2008. A Reims est igalement allustee la fabricalion des armes : ef. Nol. Dign., IX, 36, confirmé par l'inscription d'un cassidurius (I.L.T.T., 35i),

94 C.I.L., XIII, 2008.

95 A moins qu'il n'y ait ité en visile cherz son freme, vetranan retiré à Lugdumum.

96 re et entre l'origine ot la profession du personnage est fout à fait abrrant, comme me l'a fiat remarduer, avec raison, M. P.-.II. Duval.

97 Voir tes utiles remarpues de J.-P. Rry-Coguns, Forlune et rang social des gens de méliers de Tyr an BasEmpire, dans Klema, 4, 1979, p. 281-192, en particulier p. 2*2, commentaire des Inscriptions grectues el latines trouvées dans les fouilles de Tyr 1963-197.1, I. Inscriptions de la necropole, publicies par J.-P. Ris-Cogcass, dans Bull. Mus. Beyrouth, XXIX, 1977.

98 Des etudes comparatives seraicnt particulierement eclairantes, lant paraissent importantes les differences de situation des artisans et des commercants selon les epoques evidemment, mais aussi selon les regrons. Voir par ex. J.-P. Mores., Aspects de l'artisanal dans la Girande Circe remaine, dans La Magna firecia nell eta romanu. Alli del quindicesimo convegno di studi sulla Magna Grecia, Taranto, i-10 ollobre 197.5, Napoli, 1979, p. 263-32.1.

N.B. - Origine des documents : fig. 1 et 3, photos Veysseyre; fig. 2, photo Guignard-Perret; fig. 4 id 11 , photos de la Direction des Antiquités historiques Rhòne-Alpes. 